\title{
Needle-shaped amphoteric calix[4]arene as a magnetic nanocarrier for simultaneous delivery of anticancer drugs to the breast cancer cells
}

This article was published in the following Dove Medical Press journal: International Journal of Nanomedicine

\author{
Mahdi Rahimi,' Ramin \\ Karimian, ${ }^{2}$ Ehsan Bahojb \\ Noruzi, ${ }^{3}$ Khudaverdi \\ Ganbarov, ${ }^{4}$ Mojtaba Zarei,' \\ Fadhil S Kamounah, ${ }^{5}$ Bahman \\ Yousefi,' Milad Bastami, ${ }^{6}$ \\ Mehdi Yousefi, ${ }^{7}$ Hossein \\ Samadi Kafil' \\ 'Drug Applied Research Center, \\ Faculty of Medicine, Tabriz University \\ of Medical Sciences, Tabriz, Iran; \\ ${ }^{2}$ Chemical Injuries Research Center, \\ Systems biology and poisonings \\ institute, Baqiyatallah University \\ of Medical Sciences, Tehran, Iran; \\ ${ }^{3}$ Faculty of Chemistry, Department \\ of Inorganic Chemistry, University of \\ Tabriz, Tabriz, Iran; ${ }^{4}$ Department of \\ Microbiology, Baku State University, \\ Baku, Republic of Azerbaijan; \\ ${ }^{5}$ Department of Chemistry, University \\ of Copenhagen, Copenhagen. \\ Denmark; ${ }^{6}$ Biotechnology Research \\ Center, Faculty of Medicine, Tabriz \\ University of Medical Sciences, Tabriz, \\ Iran; ${ }^{7}$ Stem Cell Research Center, \\ Faculty of Medicine, Tabriz University \\ of Medical Sciences, Tabriz, Iran
}

Correspondence: Ramin Karimian Chemical Injuries Research Center, Systems Biology and Poisonings Institute, Baqiyatallah University of Medical Sciences, Mollasadra Street, Sheykh Baha'i Street, Tehran I4359I537I, Iran

Tel +989126101850

Email karimian.r@gmail.com

Hossein Samadi Kafil

Drug Applied Research Center, Faculty of Medicine, Tabriz University of Medical Sciences, Daneshgah Street, Tabriz

5।656-658II, Iran

Tel +98912 7184735

Email kafilhs@tbzmed.ac.ir
Background: Chemotherapy as an important tool for cancer treatment faces many obstacles such as multidrug resistance and adverse toxic effects on healthy tissues. Drug delivery systems has opened a new window to overcome these problems. There has been a strong interest development of new platform and system for delivof chemotherapeutic agents.

Purpose: In the present study, a green synthesis method was chosen and performed for preparation of a novel amphoteric calix[4]arene (Calix) macrocycle with low toxicity to the human body. Materials and methods: The amphoteric Calix was coated on the surface of $\mathrm{Fe}_{3} \mathrm{O}_{4}$ magnetic nanoparticles and used as a magnetic nanocarrier for simultaneous delivery of two anticancer agents, doxorubicin and methotrexate, against MCF7 cancer cells. Several chemical characterizations were done for validation of prepared nanocarrier, and in vitro loading and release studies of drugs were performed with good encapsulation efficiency.

Results: In vitro biological studies including hemolysis assay, erythrocytes sedimentation rate, red blood cells aggregation, cyto cellular internalization, and apoptosis evaluations were performed. Based on results, the developed nanocarrier has many advantages and capability for an efficient codelivery of DOX and MTX, which has a highly potent ability to kill cancer cells. Conclusion: All these results persuade us, this nanocarrier could be effectively used for cancer therapy of MCF7 breast cancer cells and is suitable for use in further animal studies in future investigations. Keywords: green synthesis, amphoteric calix[4]arene, drug delivery, combination therapy, MCF7 cells

\section{Introduction}

Macrocycle molecules are the significant class of drug-delivery vehicle like other classes of the delivery vehicle such as carbon nanotubes, nanoparticles, micelles, and dendrimers. ${ }^{1-4}$ There are a large number of macrocycles that are potentially useful for drug delivery; only five types have been studied extensively such as calixarenes, cyclodextrins, cucurbiturils, cryptands, crown ethers, and their derivatives. ${ }^{5-11}$ Construction of drug carrier from these macrocycles is of fundamental interest for applications in biotechnology and medicine. Calixarenes are a class of organic macrocyclic host molecules and have been widely studied and used in various fields since their discovery by David Gutsche around 1970 by the condensation of substituted phenols and formaldehyde in the presence of inorganic bases and more rarely acids as the catalyst. ${ }^{12}$ Since calixarenes can be modified by different functionalization at the lower and/or upper rims of the molecule, the calixarene derivatives provide useful platforms for anchoring a variety of binding units for ions and neutral molecules, and incorporating functional groups, such as ester, ether, ketone, amide, and carboxylic acid groups, exhibit excellent binding abilities. ${ }^{13-16}$ Their structures have unique three-dimensional surface, which 
made them as one of the best-known host molecules and used in the biomedical applications of supramolecular systems based on host-guest interactions. ${ }^{17,18}$ A particular interest was generated by the possibility of using calixarenes as vehicles in drug delivery and other pharmacological contexts.

Nano-sized magnetic particles $\left(\mathrm{Fe}_{3} \mathrm{O}_{4}\right)$ are recently developed and their superparamagnetic properties have opened promising new perspectives for their application in several fields such as drug-delivery systems, MRI, bioseparation, etc. ${ }^{19,20}$ For drug targeting, they can accumulate in desired locations within the body by using a permanent external magnet. ${ }^{21}$ Besides, using magnetic particles can significantly improve hyperthermia cancer treatment. This kind of therapy could raise the temperature of the target tissue to $43^{\circ} \mathrm{C}-46^{\circ} \mathrm{C}$, which makes cell more sensitive to chemotherapy and may show better results. Therefore, applying magnetic nanoparticles as nano-object in drug-delivery systems give several advantages to final drug carriers.

Developing drug carrier with either multidrug loading or conjugating for codelivery of the chemotherapeutic agents is a necessary approach to overcome the multiple drug resistance. ${ }^{22-24}$ The results and evidence revealed that anticancer drugs effects, cytotoxicity, antiproliferative activity, tumor cell apoptosis, and the low dose of anticancer drugs were increased by using drug carrier with combinational formulations. ${ }^{25}$ Improving the therapeutic index and overcoming the undesirable side effects of anticancer drugs with similar and different mechanisms of action is a promising strategy to reduce unwanted toxicity. ${ }^{26,27}$

In recent years, there has been considerable interest in using amphiphilic materials as the drug- and gene-delivery system for cancer therapy. ${ }^{28-31}$ The aim of present study is to prepare an amphoteric calix[4] arene (Calix) with cationic and anionic rim sides by a green approach. Also, the capability of this amphoteric carrier was evaluated for MCF7 cells as the human breast adenocarcinoma cell line with targeted delivery of DOX and MTX as the model anticancer drugs. The novel amphoteric nanocarrier showed negligible cytotoxicity and hemolytic effects on human red blood cells (RBCs) with good in vitro loading efficacy. Finally, to show the capability of drug-loaded nanocarrier in killing MCF cancer cells, MTT assay, cellular uptake, and Annexin V-FITC, and PI methods were used to investigate the apoptotic effect in comparison to free drugs.

\section{Materials and methods Materials}

Some starting materials such as Calix and 1-methyl3-(oxirane-2-ylmethyl)-1H-imidazole-3-ium chloride
(IL-oxirane) were synthesized according to the recent literature reports. ${ }^{3,6}$ Ferric chloride hexahydrate $\left(\mathrm{FeCl}_{3} \cdot 6 \mathrm{H}_{2} \mathrm{O}\right)$, ferrous chloride tetrahydrate $\left(\mathrm{FeCl}_{2} \cdot 4 \mathrm{H}_{2} \mathrm{O}\right)$, ammonia solution (30\%), 1-methylimidazole, epichlorohydrin, different reagents, and solvents of analytical grade were purchased from Merck Co. and used as received. Anticancer drugs were obtained from Zahravi Pharmaceutical Co. (Tabriz, Iran) and Sobhan Pharmaceuticals Co. (Tehran, Iran). RBCs stabilized with EDTA were obtained from the Iranian Blood Transfusion Institute and used with institutional bioethics approval. A purified Milli-Q deionized water (DI) was used for all experiments in this study. Furthermore, all used materials for biological protocols, including trypsin, FBS, and Roswell Park Memorial Institute 1640 growth medium (RPMI) were purchased from Gibco BRL Life Technologies. MCF7 cells as human breast cancer cells were provided by Cell Bank, Pasteur Institute of Tehran, Tehran, Iran. This study has been conducted in full accordance with the World Medical Association Declaration of Helsinki and was done after confirmation from the ethics committee of Tabriz University of Medical Sciences, Tabriz, Iran (as local institutional bioethics committee).

\section{Characterizations}

Fourier-transform infrared (FTIR) spectroscopy was used to monitor the reaction process and to determine sample's chemical structure. $\mathrm{KBr}$ method (sample to $\mathrm{KBr}$ ratio is $1: 50$ ) was used for recording the FTIR spectra on a Bruker (Shimadzu, Japan). The prepared $\mathrm{KBr}$ disk scanned against a blank $\mathrm{KBr}$ disk at wavenumbers ranging from 500 to $4,000 \mathrm{~cm}^{-1}$. The nuclear magnetic resonance (NMR) spectroscopy (Bruker Co., Billerica, MA, USA) was performed to validate the chemical structure of prepared samples. $\mathrm{D}_{2} \mathrm{O}$ was used as a solvent for calixarene derivatives. The morphology, size, and elemental percentage analysis of samples were investigated using a scanning electron microscopy (SEM; FESEM-EDX; TESCAN 5001). Before the examination, samples were mounted onto a metal stub using a carbon double-sided adhesive tape and covered with a thin layer of gold, with the aid of a direct current sputter technique (Emitechk450X; Quorum Technologies Ltd., Lewes, UK).

Furthermore, to accurately assess the modification process, elemental analysis was performed by using energy dispersive X-ray (EDX) spectroscopy. The transmission electron microscopy (TEM) analysis was used to show the size and shape of the samples (Leo 906, Zeiss, Freiburg, Germany) with an operating voltage of $100 \mathrm{kV}$. Before analysis, the suspension was ultrasonically dispersed using a probe-type ultrasonic generator $(400 \mathrm{~W})$ for 6 seconds, and the suspension was 
placed on a carbon-coated copper grid. Contact angle (CA) was used for the evaluation of hydrophilicity of the samples by sessile drop method. Two-microliter droplets of deionized water were released slowly on the coverslips containing samples pressed layer. ImageJ, an image analyzer software, was used for measuring the droplet of water on the surface of the sample. For CA analysis, the powder was pressed on a glass slide to make a uniform and smooth thin layer. The magnetic properties of samples were characterized with a vibrating sample magnetometer (VSM) (AGFM, Kashan, Iran) for determination of sample's magnetization response vs applied field at room temperature. The particle size distribution of samples was assessed using a dynamic laser-scattering technique (DLS; Zetasizer Nano ZS90; Malvern Instruments, Malvern, UK). Before analysis, a diluted suspension of the sample was prepared by PBS solution ( $\mathrm{pH} 7.4)$ to achieve the required sample density for analysis.

\section{Green synthesis of magnetic nanocarrier based on amphoteric calixarene} Synthesis of p-sulfonatocalix[4]arene (s-Calix)

This compound was synthesized using a previously reported procedure. ${ }^{32}$ Calix (1.0 g, $2.4 \mathrm{mmol}$ ) was treated with concentrated $\mathrm{H}_{2} \mathrm{SO}_{4}(10 \mathrm{~mL})$, and the mixture was stirred vigorously at $70^{\circ} \mathrm{C}$ for 3 hours. An aliquot was taken out from the solution and poured into water. The reaction progress was monitored by adding an aliquot of reaction mixture gradually into the deionized water. After completion of the reaction, no precipitate materials were detected in the mixture. The reaction mixture was allowed to cool and poured gradually into $10 \mathrm{~mL}$ deionized water. The solution was neutralized with aqueous $\mathrm{Na}_{2} \mathrm{CO}_{3}$ solution and evaporated to dryness. The residue was reprecipitated several times from water into absolute ethanol. Finally, p-sulfonatocalix[4] arene (s-Calix) was dried at $50^{\circ} \mathrm{C}$ for 24 hours, and a white crystalline solid was obtained.

\section{Synthesis of needle-shaped poly ionic liquid-grafted p-sulfonated calix[4]arene (PIL-Calix)}

Ring-opening polymerization of IL-oxirane on the surface of $\mathrm{s}$-Calix was performed by modification of a previously reported method. ${ }^{33}$ s-Calix (1.0 g, $\left.1.2 \mathrm{mmol}\right)$ was dissolved with an excess amount of IL-oxirane $(100 \mathrm{mmol})$ in deionized water, and the $\mathrm{pH}$ of the solution was adjusted to 1.0 by adding $\mathrm{HCl}$ solution. The mixture was stirred at $60^{\circ} \mathrm{C}$ under a nitrogen atmosphere for 24 hours. The resulting poly ionic liquid-grafted p-sulfonated calix[4]arene (PIL-Calix) was reprecipitated several times in ethanol to remove unreacted IL-oxirane. The obtained light brown solid (PIL-Calix) was dried in vacuum.

\section{Preparation of PIL-Calix-coated magnetic} nanoparticles (PIL-Calix/MNPs)

Briefly, PIL-Calix (1.0 g) was dissolved in $50 \mathrm{~mL}$ deionized water, and subsequently, $1.25 \mathrm{~g}$ of $\mathrm{FeCl}_{3} \cdot 6 \mathrm{H}_{2} \mathrm{O}$ and $0.5 \mathrm{~g}$ of $\mathrm{FeCl}_{2} \cdot 4 \mathrm{H}_{2} \mathrm{O}$ were added, and the solution was stirred at room temperature for 30 minutes to obtain a homogenous solution. By adding ammonia solution gradually to the above solution, $\mathrm{Fe}_{3} \mathrm{O}_{4}$ magnetic nanoparticles were formed, which modified with PIL-Calix at nanoparticles surface. The reaction mixture was stirred for 30 minutes, and the obtained PIL-Calix-coated magnetic nanoparticles (PIL-Calix/MNPs) were isolated by an external magnet and washed several times with ethanol and water. Finally, the formed PIL-Calix/MNP was dried in vacuum for further use.

\section{Blood compatibility tests}

Human whole blood was obtained from Iranian Blood Transfusion Institute stabilized with EDTA and spun at 4,000 rpm for 10 minutes at room temperature. The plasma (upper layer) was discarded, and the pellets (RBCs) were isolated. The isolated RBCs were washed three times with PBS ( $\mathrm{pH} 7.4$ ) until a clear supernatant was obtained. For the blood compatibility of PIL-Calix/MNPs, the following three tests were performed according to the procedure described below. ${ }^{34,35}$

Test A - hemolysis assay: $0.5 \mathrm{~mL}$ suspension of PILCalix/MNPs $(25,50,100,200,400$, and $800 \mu \mathrm{g} / \mathrm{mL})$ and $0.5 \mathrm{~mL}$ of diluted RBCs were mixed in a microtube and incubated at $37^{\circ} \mathrm{C}$ for 1 hour. The samples were centrifuged at 5,000 rpm for 5 minutes and the supernatant was transferred to the 96-well plates. ELISA plate reader was used to measure the released hemoglobin (at a wavelength of $540 \mathrm{~nm}$ ) and calculate the hemolysis percentage using the following formula:

$$
\text { Hemolysis rate }(\%)=\frac{\mathrm{Abs}_{\text {sample }}-\mathrm{Abs}_{\mathrm{PBS}}}{\mathrm{Abs}_{\text {water }}-\mathrm{Abs}_{\mathrm{PBS}}} \times 100
$$

Test B - erythrocytes aggregation test: after incubation of test number A, aggregation of RBCs was observed by phase contrast microscope (Nikon, YS2-T; Tokyo, Japan) with $40 \times$ magnification. Diluted RBCs treated with water and PBS were used as positive and negative controls with $100 \%$ and $0 \%$ hemolytic effects, respectively.

Test $\mathrm{C}$ - erythrocytes sedimentation rate: $2 \mathrm{~mL}$ of human blood was drawn into the Westergren tube (a narrow vertical tube) containing $0.5 \mathrm{~mL}$ sodium citrate, $2.0 \mathrm{mg}$ of PIL-Calix/ MNPs was then added, and the tube was placed in a rack in an upright position for 1 hour at room temperature. The rate 
of sedimentation is measured as the number of millimeters of clear plasma present at the top of the column after 1 hour (mm/hour).

\section{In vitro DOX and MTX loading and release studies}

In vitro drug loading and release studies were performed according to the two following procedures:

Loading step: simultaneous loading of DOX and MTX was performed as follows: PIL-Calix/MNPs (100 mg) as a nanocarrier was dispersed in PBS solution $(100 \mathrm{mg}$ ) by water bath sonication, and then MTX $(10 \mathrm{mg})$ was added to the suspension and mixed moderately with a magnetic stirrer for 24 hours. After isolation of MTX-loaded nanocarrier (MTX/ nanocarrier) and washing with PBS to remove unloaded MTX and DOX, loading was done as follows: MTX/nanocarrier was dispersed in PBS, and DOX (10 mg) was added to the suspension. The suspension was stirred at room temperature under the dark condition for 24 hours. Finally, DOX-MTXloaded magnetic nanocarrier (DOX-MTX/nanocarrier) was obtained and used in the releasing step.

Releasing step: DOX-MTX/nanocarrier $(10 \mathrm{mg})$ was added to microtube, which was categorized as follows: category 1 for $\mathrm{pH} 7.4$ and category 2 for $\mathrm{pH} 5.0$ in triplicate. Two millilters of PBS solution was added to each microtube and incubated at $37^{\circ} \mathrm{C}$. At a certain time, the microtubes were centrifuged, and the supernatant was taken for determination of released MTX and DOX by UV-Vis spectrophotometer.

\section{Cell culture}

MCF7 cells as the human breast adenocarcinoma cell line were used in this study which was obtained from the National Cell Bank of Iran (Pasteur Institute of Iran, Tehran, Iran). The cells were cultured with RPMI 1640 medium (supplemented with $10 \%(\mathrm{v} / \mathrm{v}) \mathrm{FBS}, 1 \%$ antibiotics including penicillin and streptomycin with a concentration of $50 \mathrm{IU} / \mathrm{mL}$ and $50 \mu \mathrm{g} / \mathrm{mL}$, respectively). After cell culturing, the flask containing cells were incubated under a fully humidified atmosphere at $37^{\circ} \mathrm{C}$ with $5 \% \mathrm{CO}_{2}$, and growth medium must be renewed two to three times per week until the cells reached $90 \%$ confluency. After sufficient PBS washing, the cells were detached with $0.25 \%$ trypsin in PBS (pH 7.4) and centrifuged at $1,500 \mathrm{rpm}$ for 5 minutes. ${ }^{36}$ Subsequently, the cells were dispersed in growth medium and used for seeding in further in vitro assays.

\section{Cellular uptake}

MCF7 cells were seeded in 6-well plates for quantification of uptake at a density of $5 \times 10^{4}$ and $5 \times 10^{5}$ cells per well for cellular uptake by fluorescent microscopy and flow cytometry assays, respectively. ${ }^{2}$ After incubation of cells for 24 hours, the culture media of 6-well plates were replaced with free media. Then, the cells were treated with different sample suspensions including DOX-labeled nanocarrier (at a concentration of $10 \mu \mathrm{g} / \mathrm{mL}$ in PBS), and the cells with no treatment considered as a control. In the end, two following regular procedures were performed.

Cellular uptake by fluorescent microscopy: after incubation for 1, 2, and 3 hours, the cells were washed three times with PBS and fixed by formaldehyde ( $4 \% \mathrm{w} / \mathrm{v})$. The coverslip was taken onto a glass microscope slide, and DOX/nanocarrier uptake was observed by using a fluorescence microscope (Olympus Microscope Bh2-RFCA, Tokyo, Japan).

Cellular uptake by flow cytometry: after incubation for 1,2 , and 3 hours, the cells were trypsinized and collected for determination of DOX/nanocarrier uptake inside the cells via fluorescence intensity using a FACSCalibur flow cytometer (Becton Dickinson Immunocytometry Systems, San Jose, CA, USA).

\section{Cytotoxicity assay}

The MCF7 cells at the concentration of $10 \times 10^{3}$ cells per well were seeded in 96-well plates and incubated under $5 \% \mathrm{CO}_{2}$ atmosphere for overnight at $37^{\circ} \mathrm{C}$ attached at the bottom of each well. For in vitro cytotoxicity evolutions, following procedure was performed. Briefly, the seeded cells were treated with different samples including (blank nanocarrier, DOX-MTX/nanocarrier, and DOX-MTX) at various concentrations in RPMI medium and incubated for 48 hours. After washing several times with PBS, MTT assays were performed to determine the cell viability according to the reported procedure. The absorbance of each well was measured at $570 \mathrm{~nm}$ with an ELISA plate reader.

\section{DAPI staining}

The apoptotic effects of samples on MCF7 cells were examined by DAPI staining as a routine microscopic analysis. ${ }^{37}$ Briefly, in 6-well plates containing sterile coverslips, $5 \times 10^{4}$ MCF7 cells were seeded and allowed attaching at the bottom of each well at $37^{\circ} \mathrm{C}$ for overnight. The cells were treated with blank nanocarrier, DOX-MTX/nanocarrier, and DOXMTX at the concentration of $\mathrm{IC}_{50}$. The cells with no treatment were considered as a negative control. The 6-well plates were kept in an incubator and incubated for 48 hours. The wells were washed three times with PBS and fixed with $4 \%$ formaldehyde at ambient temperature for 15 minutes. Then cells were permeabilized by adding Triton X-100 $(0.1 \% \mathrm{w} / \mathrm{v})$ and incubated for 5 minutes. Ultimately, cells were stained by 
using DAPI solution (200 ng/mL), and DNA condensation and fragmentation in apoptotic cells were evaluated under a fluorescence microscope (Olympus Microscope Bh2RFCA, Japan).

\section{Apoptosis assay by flow cytometry}

Apoptotic effects of different formulations were measured by ApoFlowEx ${ }^{\circledR}$ FITC Kit (EXBIO Diagnostics, Vastec, Czech Republic). MCF7 cancer cells at the density of $5 \times 10^{5}$ cells per well were seeded in 6-well plates and incubated for 24 hours attached at the bottom of the wells. Then, the cells were treated with blank nanocarrier, free DOX-MTX, and DOX-MTX/nanocarrier. Cells receiving only fresh medium were considered as control group. After incubation for 48 hours, cells were trypsinized, washed twice with cold PBS, resuspended in $1 \times$ binding buffer $(500 \mu \mathrm{L})$, added $5 \mu \mathrm{L}$ of Annexin V-FITC and $5 \mu \mathrm{L}$ of propidium iodide, and incubated for further 15 minutes in dark. Then flow cytometry analysis was performed by flow cytometer (FACSCalibur; BD Biosciences, San Jose, CA, USA).

\section{Statistical analysis}

All data were reported as mean \pm SD of three replicates. The ANOVA was used for statistical analysis. The $P$-values $<0.05$ were considered significant.

\section{Results and discussion Synthesis}

As shown in Figure 1A, Calix is a cavity-shaped cyclic molecule with four phenol units linked with methylene bridges at ortho positions. Calix has four tertiary butyl (t-butyl) functional groups at the para position of hydroxyl groups, which render them very hydrophobic. It has a crystalline structure under the microscope with a very light yellow color. After treating with concentrated sulfuric acid, the t-butyl groups at para position were substituted with sulfonate $\left(-\mathrm{SO}_{3} \mathrm{H}\right)$ hydrophilic groups, and the physicochemical characteristics of the resulting compound were changed. The crystalline form of sulfonate calixarene under the microscope showed the same appearance as Calix (Figure 1B). The sulfonate groups act as anionic active sites for loading
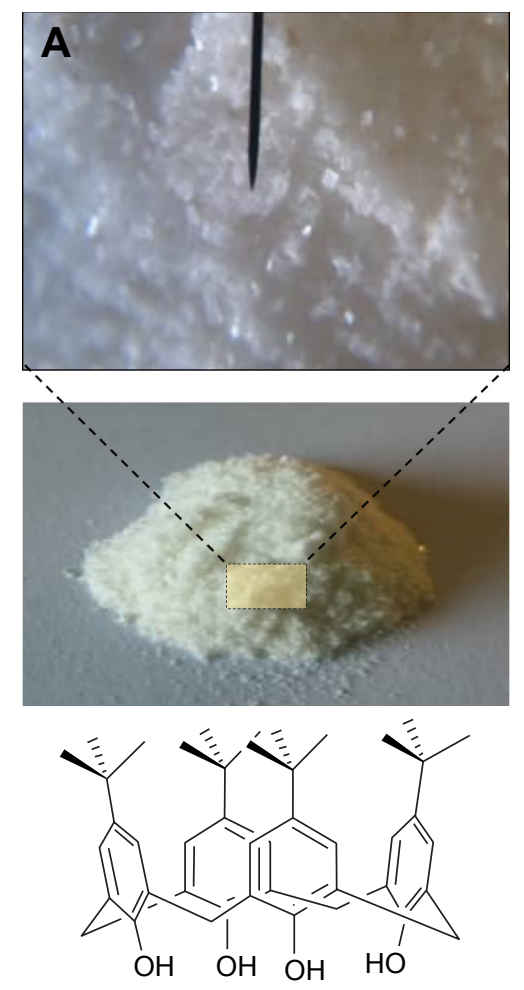
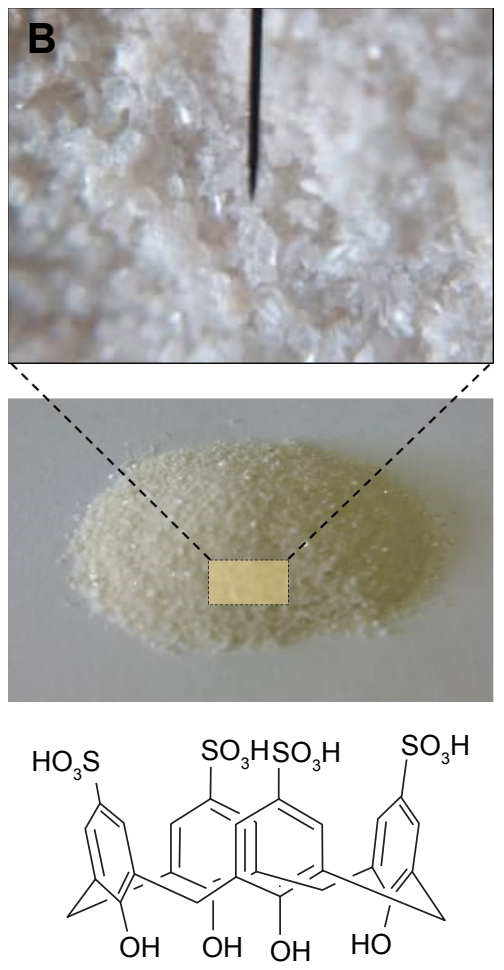
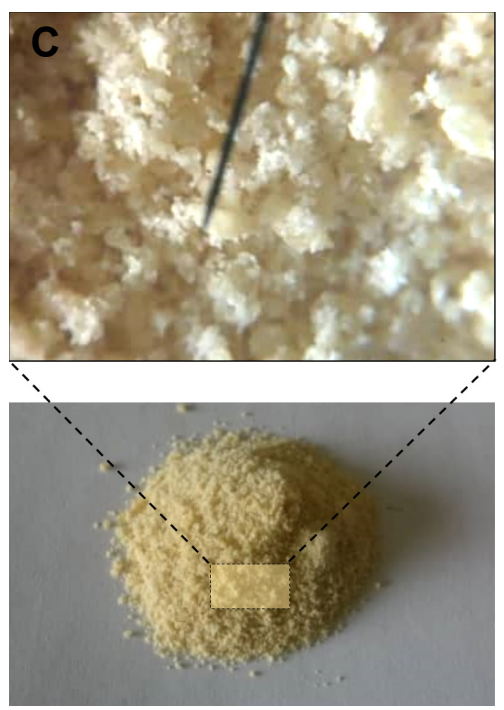

$\mathrm{HO}_{3} \mathrm{~S}$
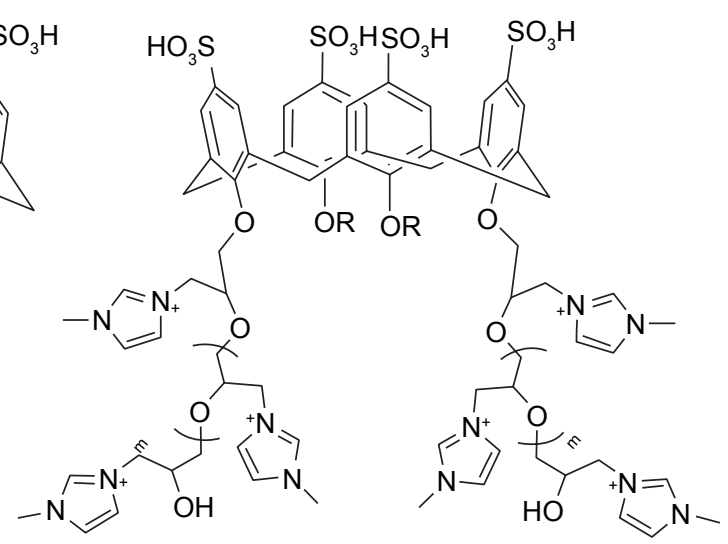

Figure I Chemical structure, appearance, and crystalline structure of Calix, s-Calix, and PIL-Calix molecules.

Notes: Calix (A), s-Calix (B), PIL-Calix molecules (C).

Abbreviations: Calix, calix[4]arene; PIL-Calix, poly ionic liquid-grafted p-sulfonated calix[4]arene; s-Calix, p-sulfonatocalix[4]arene. 
A
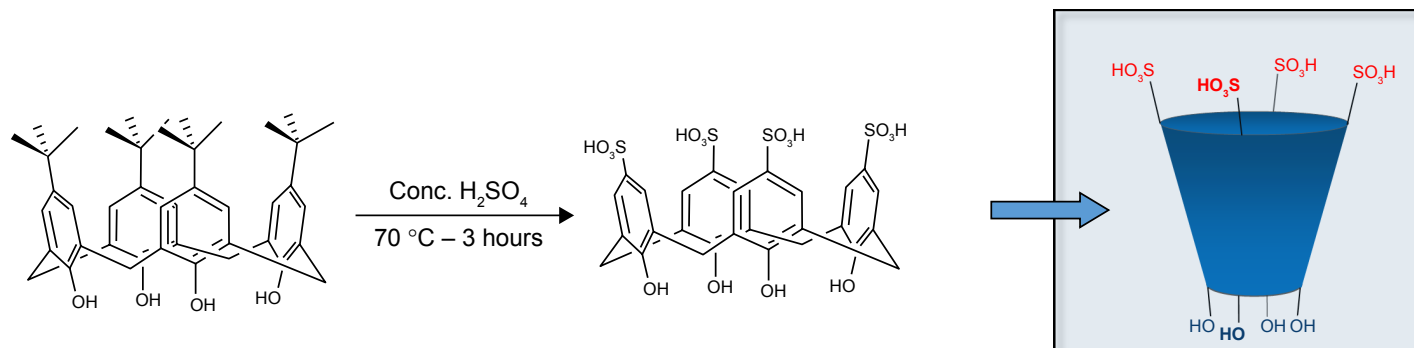

B

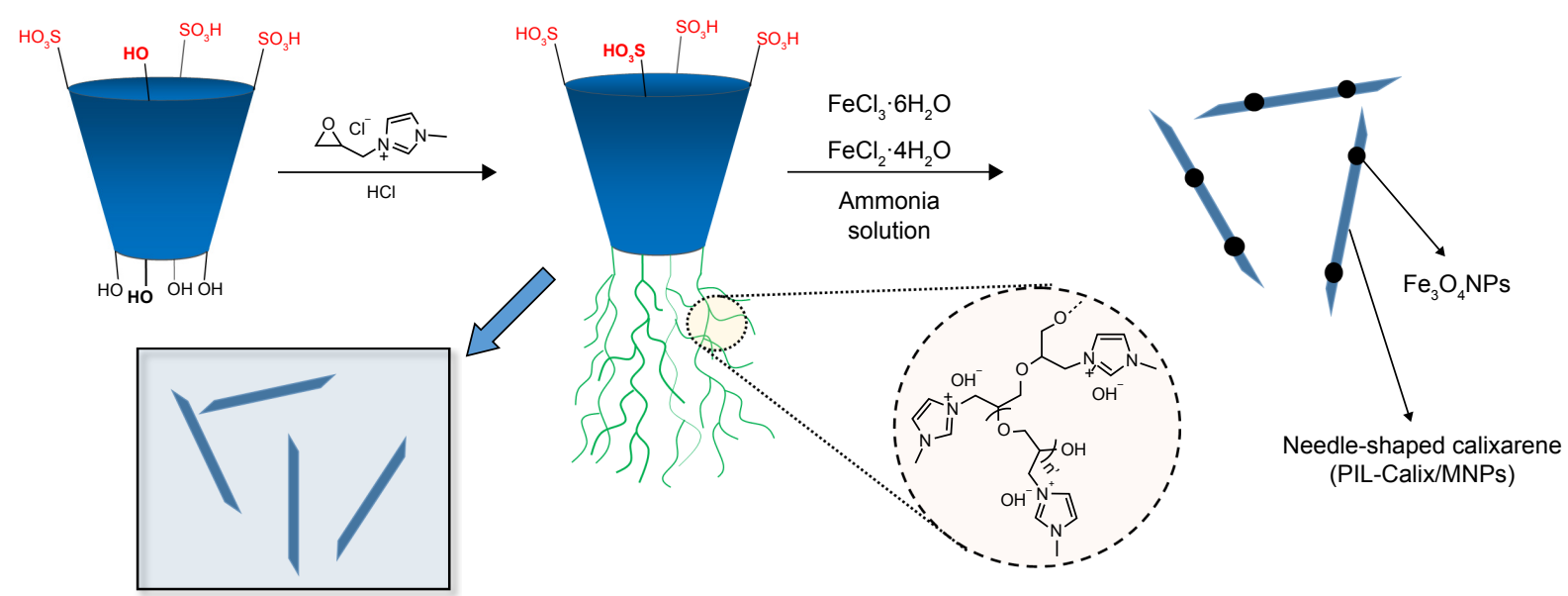

Figure 2 (A) Preparation of p-sulfonatocalix[4]arene (s-Calix) from 4-tert-butylcalix[4]arene (Calix). (B) Preparation of needle-shaped poly ionic liquid-grafted p-sulfonated calix[4]arene (PIL-Calix) via ring-opening polymerization of IL oxirane (step I) and preparation of PIL-Calix-coated magnetic nanoparticles (PIL-Calix/MNPs) as a magnetic nanocarrier (step 2).

doxorubicin drug. On the other hand, to create cationic active sites on these macrocycles for loading MTX drug, ionic liquid polymeric chains were attached to the other side of macrocycles causing amphoteric Calix. The resulting calixarene has different appearance without crystalline structure (Figure 1C). All reaction processes were performed in green approach with no toxic solvents, making the designed nanocarrier very safe for use in the biomedical applications (Figure 2).

\section{Characterizations}

Before performing biological assays to show the safety of nanocarrier and its capability in combinational delivery of anticancer drugs in drug-delivery systems, all materials were characterized by several techniques including FTIR, NMR, differential scanning calorimetry (DSC), SEM, EDX, TEM, VSM, DLS, and CA. In the following section, each analysis was discussed as well.

All samples were characterized by FTIR and ${ }^{1} \mathrm{H}$ NMR spectroscopies to determine the functional groups and chemical structure of the samples. As shown in Figure 3, the FTIR spectra of Calix, s-Calix, PIL-Calix, and PIL-Calix/MNPs were taken. The FTIR characteristic absorbance and ${ }^{1} \mathrm{H}$ NMR chemical shifts of the samples are given below.

Calix: the characteristic absorption peaks at 670 , $1,242,1,481,2,956,3,049$, and $3,201 \mathrm{~cm}^{-1}$ are attributed to

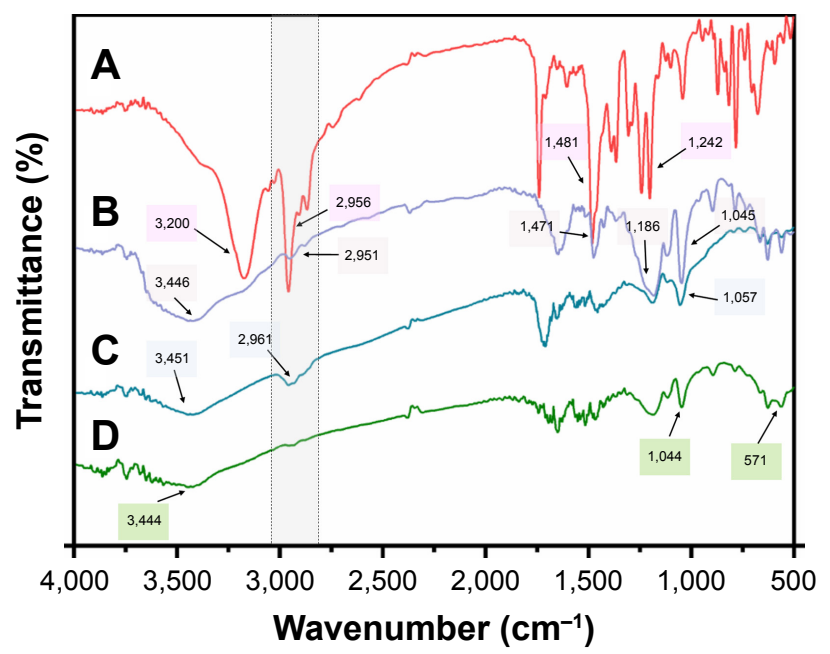

Figure 3 FTIR spectra of Calix (A), s-Calix (B), PIL-Calix (C), and PIL-Calix/MNPs (D) at wavenumber range of $500-4,000 \mathrm{~cm}^{-1}$

Abbreviations: Calix, calix[4]arene; PIL-Calix, poly ionic liquid-grafted p-sulfonated calix[4]arene; PIL-Calix/MNPs, PIL-Calix-coated magnetic nanoparticles; s-Calix, p-sulfonatocalix[4]arene. 
the $=\mathrm{C}$-hour bending, $\mathrm{C}-\mathrm{O}$ stretching, $\mathrm{C}=\mathrm{C}$ stretching, aliphatic $\mathrm{C}-\mathrm{H}$ stretching, aromatic $\mathrm{C}-\mathrm{H}$ stretching, and $\mathrm{O}-\mathrm{H}$ stretching bonds, respectively. Due to the presence of the t-butyl group substituted at the para position of phenol, the intensity of the peaks at around 2,956 is high.

${ }^{1} \mathrm{H}-\mathrm{NMR}\left(400 \mathrm{MHz}, \mathrm{CDCl}_{3}, \delta \mathrm{ppm}\right): 10.3(\mathrm{OH}, \mathrm{s}, 4 \mathrm{H})$, 7.05 (Ar-H, s, $8 \mathrm{H}), 4.25\left(\mathrm{CH}_{2}\right.$, d, $\left.4 \mathrm{H}\right), 3.49\left(\mathrm{CH}_{2}, \mathrm{~d}, 4 \mathrm{H}\right)$, and $1.21(\mathrm{t}-\mathrm{Bu}, \mathrm{s}, 36 \mathrm{H})$.

s-Calix: the characteristic peaks at $669,1,045,1,186$, $1,213,1,471,2,954,3,190$, and $3,446 \mathrm{~cm}^{-1}$ are assigned to the $=\mathrm{C}-\mathrm{H}$ bending, asymmetric stretching vibration of $\mathrm{S}-\mathrm{O}$, $\mathrm{C}-\mathrm{O}$ stretching, splitting of the $\mathrm{S}=\mathrm{O}$ group into symmetric and asymmetric stretching vibrations, $\mathrm{C}=\mathrm{C}$ stretching, aliphatic $\mathrm{C}-\mathrm{H}$ stretching, aromatic $\mathrm{C}-\mathrm{H}$ stretching, and $\mathrm{O}-\mathrm{H}$ stretching bonds, respectively.

${ }^{1} \mathrm{H}-\mathrm{NMR}\left(400 \mathrm{MHz}, \mathrm{D}_{2} \mathrm{O}, \delta\right.$ ppm): 7.60 (ArH, s, $8 \mathrm{H}$ ) and $3.84\left(\mathrm{CH}_{2}, \mathrm{~S}, 8 \mathrm{H}\right)$.

PIL-Calix: as the PIL-Calix has polymeric chains on its structure with several methylene groups $\left(-\mathrm{CH}_{2}-\right),-\mathrm{C}-\mathrm{N}=$, $-\mathrm{C}=\mathrm{N}-$, and $-\mathrm{C}-\mathrm{OH}$, it was expected that some new peaks or shifting absorption peaks were observed. Regarding the formation of many methylene groups in the polymeric chains, the absorption peaks at $2,961 \mathrm{~cm}^{-1}$ were enhanced in comparison to the s-Calix. The characteristic peak of $-\mathrm{C}=\mathrm{N}-$ sections was appeared at $1,680 \mathrm{~cm}^{-1}$, whereas the peak of $-\mathrm{C}-\mathrm{N}=$ was not appeared clearly because of its low intensity. Identical peaks in both s-Calix and PIL-Calix appeared at the same range of wave number with slight shifting.

${ }^{1} \mathrm{H}-\mathrm{NMR}\left(400 \mathrm{MHz}, \mathrm{D}_{2} \mathrm{O}, \delta \mathrm{ppm}\right): 8.58(\mathrm{~N}=\mathrm{CH}, \mathrm{s}), 7.60$ (ArH, s, 8 H), 7.32-6.94 (NCH, m), 4.18 (NCH3, s), 3.84 (CH2, S, 8 H), and 3.5-3.22 (OCH2CHCH2N, m).

PIL-Calix/MNPs: after surface modification of magnetic nanoparticles $\left(\mathrm{Fe}_{3} \mathrm{O}_{4}\right)$ with PIL-Calix, the characteristic peaks of both $\mathrm{Fe}_{3} \mathrm{O}_{4}$ and PIL-Calix were observed. $\mathrm{Fe}_{3} \mathrm{O}_{4}$ has a bulk network with two clear peaks at 573 and $3,458 \mathrm{~cm}^{-1}$ for $\mathrm{Fe}-\mathrm{O}$ and $\mathrm{O}-\mathrm{H}$. By following the PIL-Calix/MNPs spectrum, the characteristic peaks of PIL-Calix were also found with low intensity. All observations and results validated our preparation process.

DSC is a technique for analyzing the thermal behavior of the sample according to the amount of heat required to increase the temperature. The DSC graph is depicted based on heat flow vs temperature. The DSC thermographs of Calix, s-Calix, and PIL-Calix are shown in Figure 4. Calix showed a uniform curve with no exothermic and endothermic peaks in the analysis temperature range, and it has no phase transition with a melting point above this range (melting point $>300^{\circ} \mathrm{C}$ ). However, s-Calix has a sharp exothermic

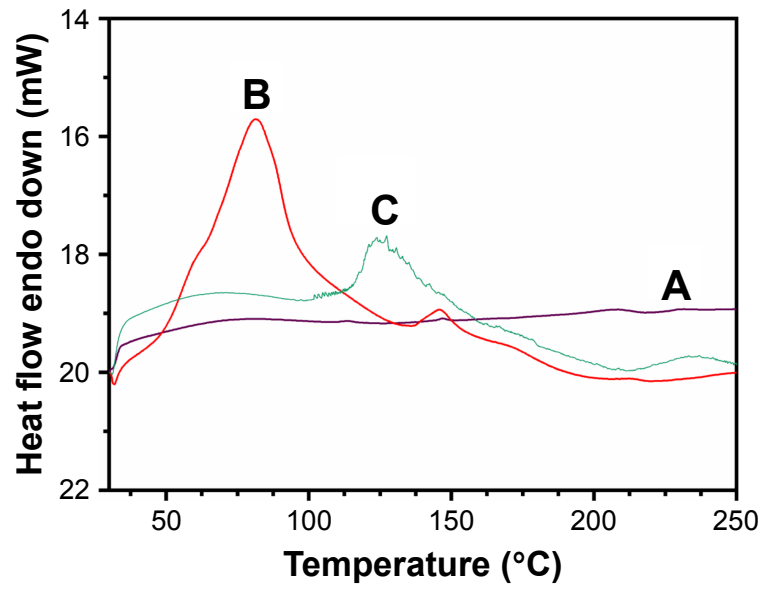

Figure 4 DSC thermographs of Calix (A), s-Calix (B), and PIL-Calix (C) from $30^{\circ} \mathrm{C}$ to $250^{\circ} \mathrm{C}$ at a heating rate of $5^{\circ} \mathrm{C} / \mathrm{min}$ under a flowing nitrogen atmosphere. Abbreviations: Calix, calix[4]arene; DSC, differential scanning calorimetry; PIL-Calix, poly ionic liquid-grafted p-sulfonated calix[4]arene; s-Calix, p-sulfonatocalix[4]arene.

peak in the temperature range of $50^{\circ} \mathrm{C}-100^{\circ} \mathrm{C}$, which is attributed to the recrystallization of molecules with high heat releasing rate. At this point, the fragments may obtain enough freedom of motion to arrange themselves into a crystalline form spontaneously. Due to the presence of polymeric branches on the PIL-Calix, it was expected that the recrystallization temperature shifted to the higher temperature because the polymeric chains prevent rearrangement of the molecules in lower temperature. By observing the DSC graph of PIL-Calix, the height of the curve was more depressed than unmodified s-Calix and can be concluded that low exothermic heat was released. All results confirmed that the modification in each step was successful.

The water CA is a measure of the wettability of the solid surface. If the solid surface is highly hydrophilic, water droplet is strongly attracted, and then the water drop will completely spread out with $\mathrm{CA}(\theta)$ of $0^{\circ}$. In general, water CA lower and more substantial than $90^{\circ}$ is considered hydrophilic and hydrophobic solid surfaces, respectively. Regarding this, an appropriate amount of each sample was placed on a glass slide, and another glass slide was fixed on the sample. The glass slides were placed in the pressure instrument, and the sample was pressed slowly. The obtained disk on glass slide had a homogenous surface. Given that the conditions are the same, the results could be compared to each other. Since Calix contains four t-butyl groups, which cause it highly hydrophobic, its water CA value could not be measured by instrument due to the high hydrophobicity and escaping from the water. Figure 5 showed that the droplet could not separate and stand on the surface. (This picture was snapshotted from the video of the measurement process.) After exchanging 
A
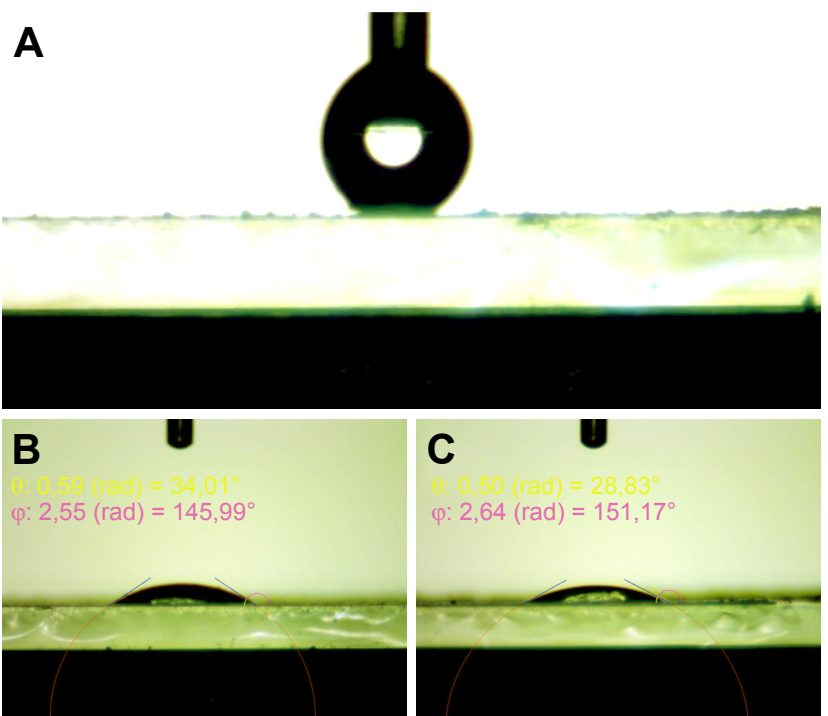

Figure 5 Water drop contact angle for hydrophobic Calix (A) and hydrophilic s-Calix (B), and PIL-Calix (C) samples.

Abbreviations: Calix, calix[4]arene; PIL-Calix, poly ionic liquid-grafted p-sulfonated calix[4]arene; s-Calix, p-sulfonatocalix[4]arene. the t-butyl groups with sulfonate $\left(-\mathrm{SO}_{3} \mathrm{H}\right)$ group to yield s-Calix, it was expected that the hydrophilicity enhanced after substitution of t-butyl with sulfonate and the amount of $\theta$ decrease to lower degree (34.01). The hydrophilicity of PIL-Calix was also evaluated and results showed that water droplet could spread out easily on the surface of film with $\theta$ value of 28.83. All samples were tested in triplicate at three different areas, and obtained midpoint results were reported. Overall, the results data confirmed that the present modification process was done.

The surface morphology of Calix, s-Calix, PIL-Calix, and PIL-Calix/MNPs was studied by SEM analysis and illustrated in Figure 6. Calix has a solid structure with finger-like beads and smooth surface. The s-Calix also has finger-like beads. However, its morphology was changed to rough form with several fractions on it. Further evaluation revealed that each bead was changed to multilayer structure. Surface morphology of PIL-Calix revealed that the structure of s-Calix was
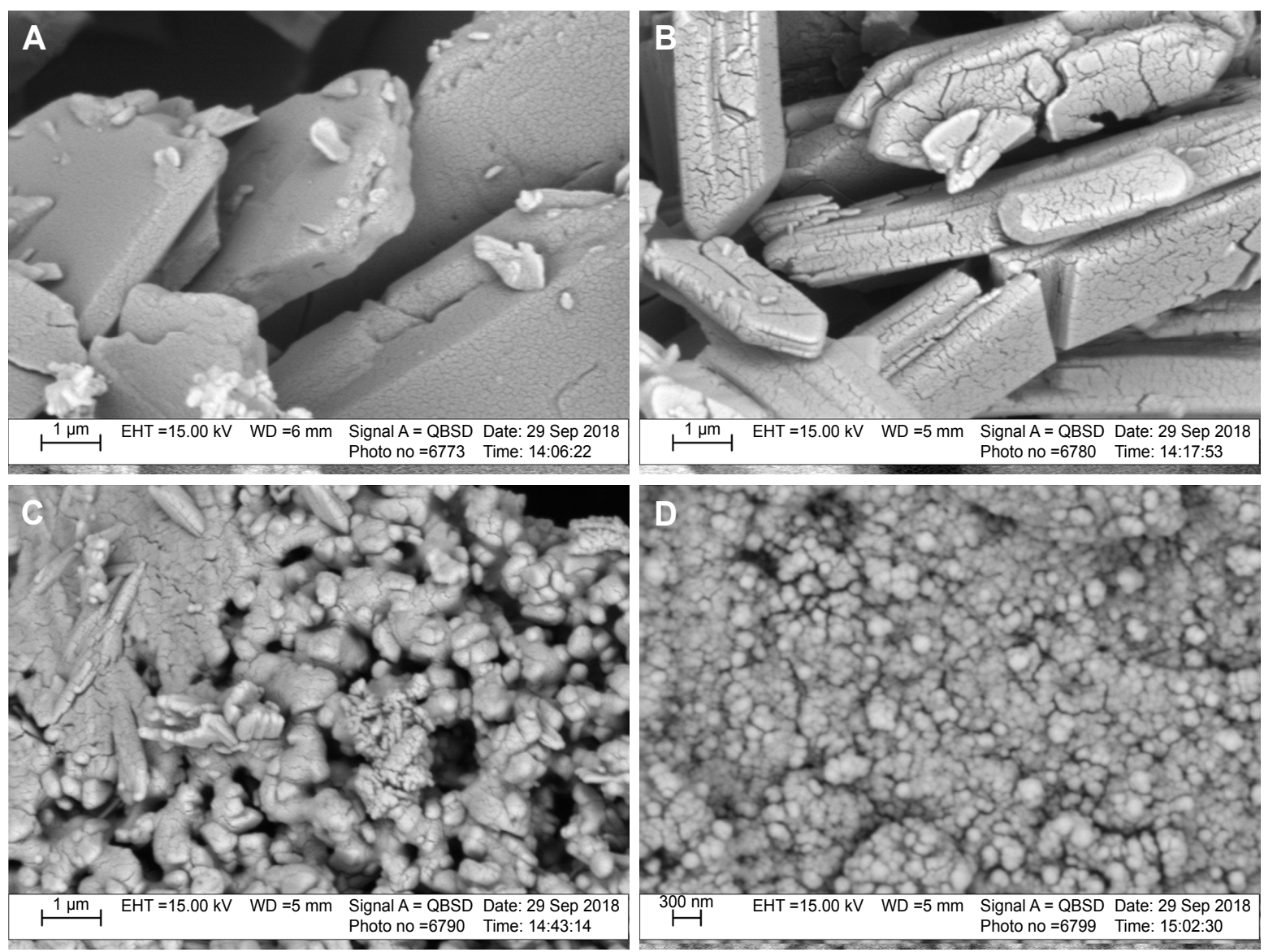

Figure 6 Surface morphology and size of Calix (A), s-Calix (B), PIL-Calix (C), and PIL-Calix/MNPs (D) by SEM technique at the scale of I $\mu \mathrm{m}, \mathrm{I} \mu \mathrm{m}$, I $\mu \mathrm{m}$, and $300 \mathrm{~nm}$, respectively.

Abbreviations: Calix, calix[4]arene; PIL-Calix, poly ionic liquid-grafted p-sulfonated calix[4]arene; PIL-Calix/MNPs, PIL-Calix-coated magnetic nanoparticles; s-Calix, p-sulfonatocalix[4]arene; SEM, scanning electron microscopy. 
A

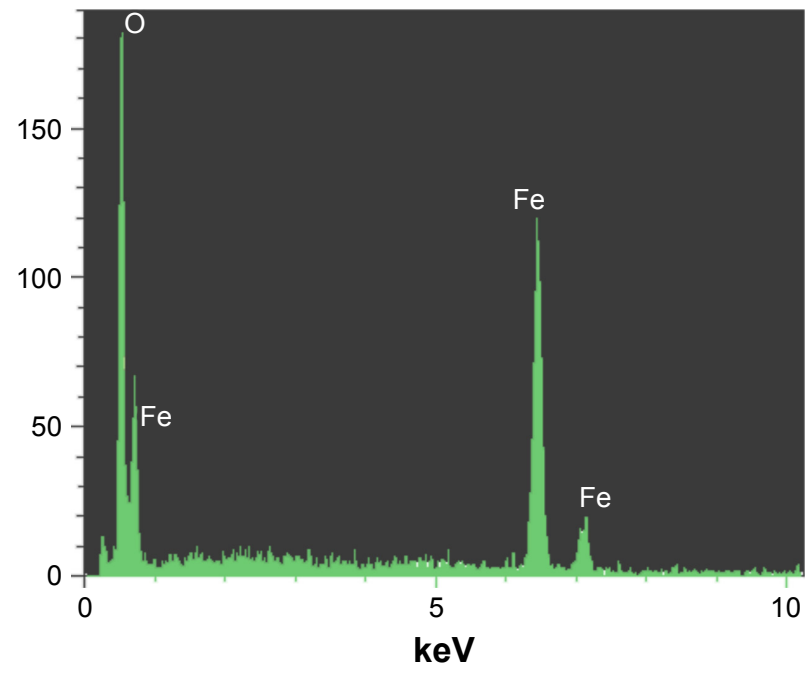

\section{B}

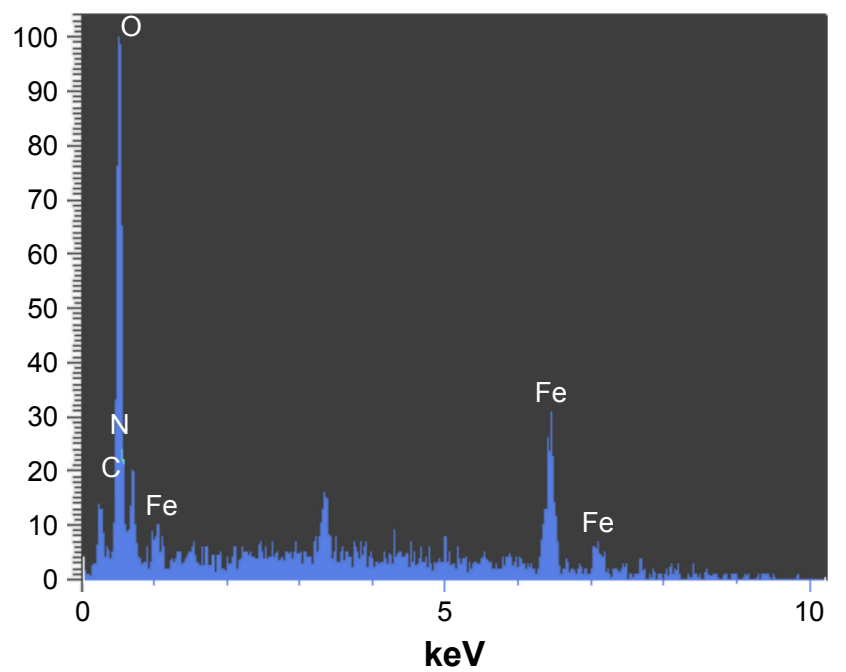

Figure 7 The EDS spectra of $\mathrm{Fe}_{3} \mathrm{O}_{4}(\mathbf{A})$ and PIL-Calix/MNPs (B) for the estimation of the elemental analysis.

Abbreviations: EDS, energy dispersive X-ray spectroscopy; PIL-Calix/MNPs, PIL-Calix-coated magnetic nanoparticles.

completely altered to various and specific shapes, which will facilitate the attachment of drug molecules onto the surface and PIL-Calix cavities. After surface modification of magnetic nanoparticle with PIL-Calix, spherical particles with nanosize around 40-50 nm were formed. The SEM image demonstrated that prepared PIL-Calix/MNPs tended to aggregate in aqueous solution because of the small size effect caused by hydrophilic units of the compound on the $\mathrm{Fe}_{3} \mathrm{O}_{4}$ nanoparticles. All observations confirmed that surface modification processes were performed successfully.

EDX spectroscopy (EDS) or EDX is an analytical technique for chemical characterization and determination of the elemental analysis. EDS can be used to estimate chemical elements relative abundance. Several factors (such as overlapping X-ray emission peaks, the nature of the sample, etc) affected the accuracy of the sample compositions. $\mathrm{Fe}_{3} \mathrm{O}_{4}$ NPs were synthesized and used as a control. As seen in the EDS spectrum (Figure 7), the atomic percentage of the iron atom $(\mathrm{Fe})$ is $40.17 \% \pm 6.65 \%$ with two characteristic peaks at 0.705 and $6.398 \mathrm{keV}$. Also, the oxygen atom (O) has $59.83 \% \pm 5.98 \%$ atomic percentage with a characteristic peak at $0.525 \mathrm{keV}$. After preparation of PIL-Calix/MNPs by in situ synthesis of $\mathrm{Fe}_{3} \mathrm{O}_{4}$ NPs in PIL-Calix solution, the EDS analysis was taken, and the atomic percentages were reported. As seen in the spectrum, two new peaks of carbon $(\mathrm{C})$ and nitrogen $(\mathrm{N})$ were appeared at around 0.277 and $0.392 \mathrm{keV}$, respectively, with atomic percentage of $22.28 \% \pm 6.23 \%$ and $3.64 \% \pm 0.87 \%$. As X-ray emission peaks of $\mathrm{N}$ and $\mathrm{O}$ elements appears close to each other, their peaks were overlapped with each other. By appearance of these elements, the intensity of $\mathrm{Fe}$ and $\mathrm{O}$ elements was decreased. All results validated the successful preparation of PIL-Calix/MNPs. The elemental analysis data were summarized in Table 1.

To receive more direct information on the particle size and morphology, TEM micrographs of PIL-Calix/MNPs were investigated. As shown in Figure 8, TEM image shows a difference in morphology. Some needle- and rod-shaped calixarene-doped magnetic nanoparticles were also observed, where magnetic nanoparticles formed on the surface of PILCalix needles and rods. Many dense aggregations of pure $\mathrm{Fe}_{3} \mathrm{O}_{4}$ magnetic nanoparticles were observed, due to the lack of repulsive force. After PIL-Calix immobilization, the dispersion of particles was improved as well, which can easily be explained by the electrostatic repulsion force and steric hindrance between the Calix and the surface of $\mathrm{Fe}_{3} \mathrm{O}_{4}$ nanoparticles.

To measure magnetic properties of magnetic materials, VSM was used, and the magnetization value $(\mathrm{emu} / \mathrm{g})$ vs applied field (Oe) was determined at room temperature

Table I Elemental analysis data based on weight percentage (W\%) and atomic percentage (A\%)

\begin{tabular}{l|l|l|l|l}
\hline Element & \multicolumn{2}{|l|}{$\mathrm{Fe}_{3} \mathbf{O}_{4}$} & \multicolumn{2}{l}{ PIL-Calix/MNPs } \\
\hline $\mathrm{Fe}$ & $70.09 \pm 8.26$ & $40.17 \pm 6.65$ & $23.79 \pm 3.21$ & $7.7 \mathrm{I} \pm 1.29$ \\
$\mathrm{O}$ & $29.91 \pm 5.22$ & $59.83 \pm 5.98$ & $58.62 \pm 9.01$ & $66.36 \pm 9.74$ \\
$\mathrm{C}$ & - & - & $14.77 \pm 2.09$ & $22.28 \pm 6.23$ \\
$\mathrm{~N}$ & - & - & $2.80 \pm 0.88$ & $3.64 \pm 0.87$ \\
Total & 100.00 & 100.00 & 100.00 & 100.00 \\
\hline
\end{tabular}

Abbreviation: PIL-Calix/MNPs, PIL-Calix-coated magnetic nanoparticles. 


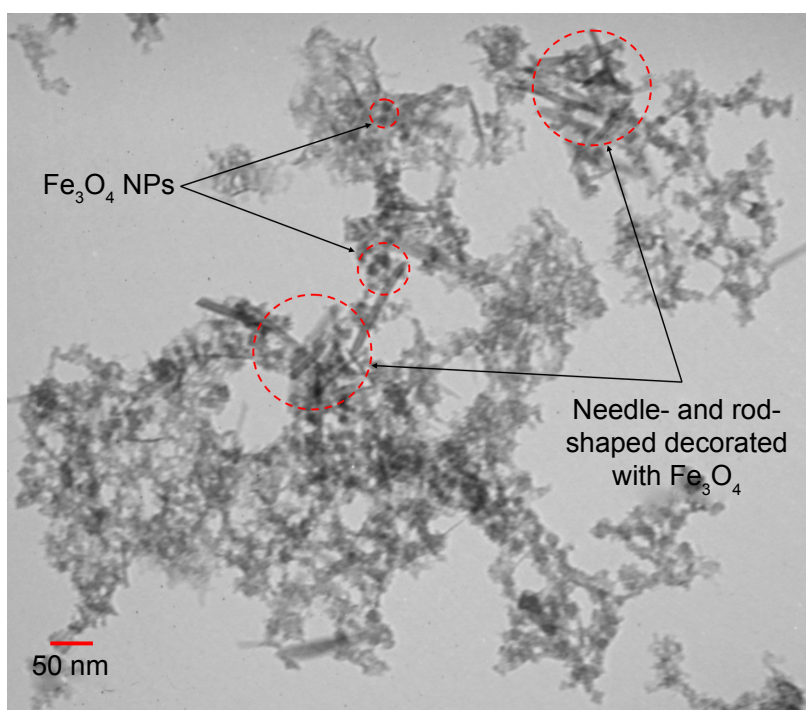

Figure 8 TEM micrograph of PIL-Calix/MNPs with a needle-shaped structure. Abbreviations: PIL-Calix/MNPs, PIL-Calix-coated magnetic nanoparticles; TEM, transmission electron microscopy.

(Figure 9). The prepared nanocarrier displayed saturation magnetization equal to about $62 \mathrm{emu} / \mathrm{g}$, which has lower amount than $\mathrm{Fe}_{3} \mathrm{O}_{4}$ NPs as a control (with magnetization value of $74 \mathrm{emu} / \mathrm{g}$ ), and it is attributed to the presence of a polymeric branch of PIL-Calix on the magnetic core. The $\mathrm{S}$ shape of the magnetic nanocarrier diagram exhibited zero coercivity and permanence, thus indicating its superparamagnetic feature. Therefore, this property of nanocarrier
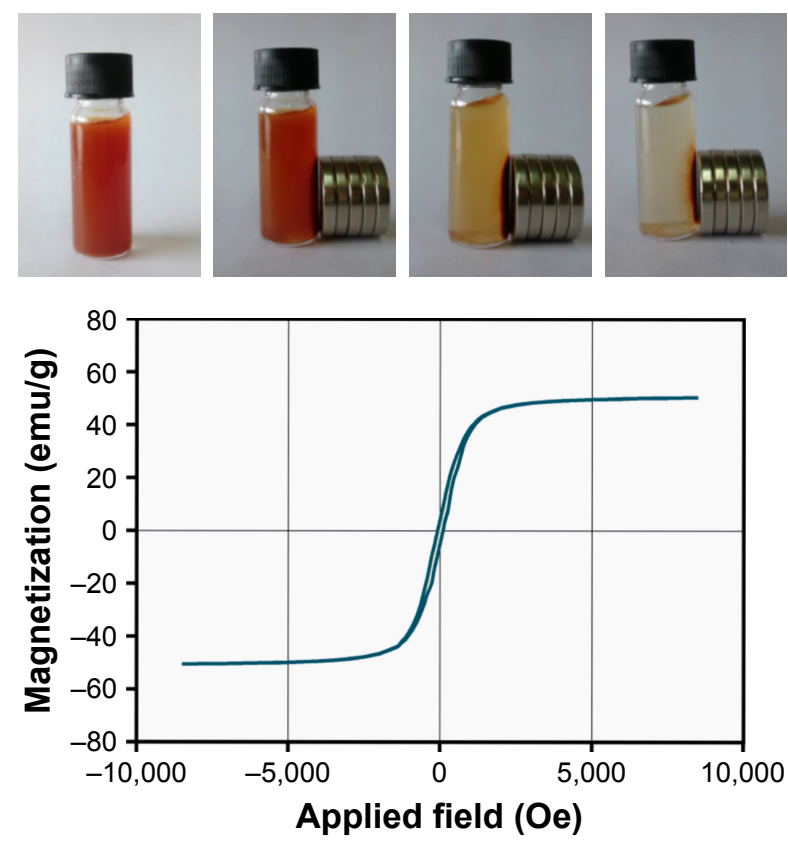

Figure 9 VSM analysis was applied to measure the magnetic properties of prepared nanocarrier at room temperature.

Abbreviation: VSM, vibrating sample magnetometer. could carry chemotherapeutic agents to the target cells by using a permanent external magnet. After using an external magnet, the separation time for collecting magnetic nanocarrier beside the container is about 20 seconds. All results confirmed that the nanocarrier is suitable for use in the pharmaceutical field.

Despite the size of the magnetic nanoparticles was observed and calculated by SEM analysis. Particle size can be determined by measuring the random changes in the intensity of light scattered from a suspension. DLS is a proper technique that is most commonly used for analyzing nanoparticle size distribution. However, this technique was performed in an aqueous solution to enable particles to interact with each other freely, which caused aggregation. As seen from the DLS graph of prepared nanocarrier (Figure 10), the size distribution is around $210 \mathrm{~nm}$.

\section{Stability of nanocarrier in the physiological condition}

The colloidal behavior of PIL-Calix/MNPs as a magnetic nanocarrier was investigated, and the results discussed as follows. The colloidal stability of magnetic nanoparticles $\left(\mathrm{Fe}_{3} \mathrm{O}_{4} \mathrm{NPs}\right)$ in an aqueous solution depends on the environmental parameters and their intrinsic magnetic properties. However, the surface modification of nanoparticles could enhance its stability, bioavailability, and toxicity. Several parameters (such as $\mathrm{pH}$, ionic strength, and plasma protein concentration) were affected by the properties of magnetic nanoparticles in physiological condition. Therefore, we used a common culture medium (RPMI 1640), which contained mono and divalent ions $\left(\mathrm{Na}^{+}, \mathrm{Mg}^{2+}\right.$, and $\left.\mathrm{Ca}^{2+}\right)$, and FBS was also added to the culture medium for cell culturing and it provided the simulated condition of the physiological body. As the stability could be affected by altering nanoparticles concentration, two different concentrations with the low and high amount of magnetic nanocarriers $(0.1 \%$ and $1 \%)$ were evaluated. The results showed that magnetic nanocarrier stability in both concentrations has not changed after incubation in culture medium for 10 days and no significant macroscopic changes were observed. This demonstrates that the stability of magnetic nanocarrier in physiological conditions is a complex function of nanoparticles surface characteristics and components of the surrounding medium. The results confirmed that the suspension has good stability with low rate sedimentation, which breaks down the aggregation by sonication or stirring. To show the diffusing capability of nanocarrier, a droplet of nanocarrier suspension was added to the simulated physiological condition medium with PBS, and 


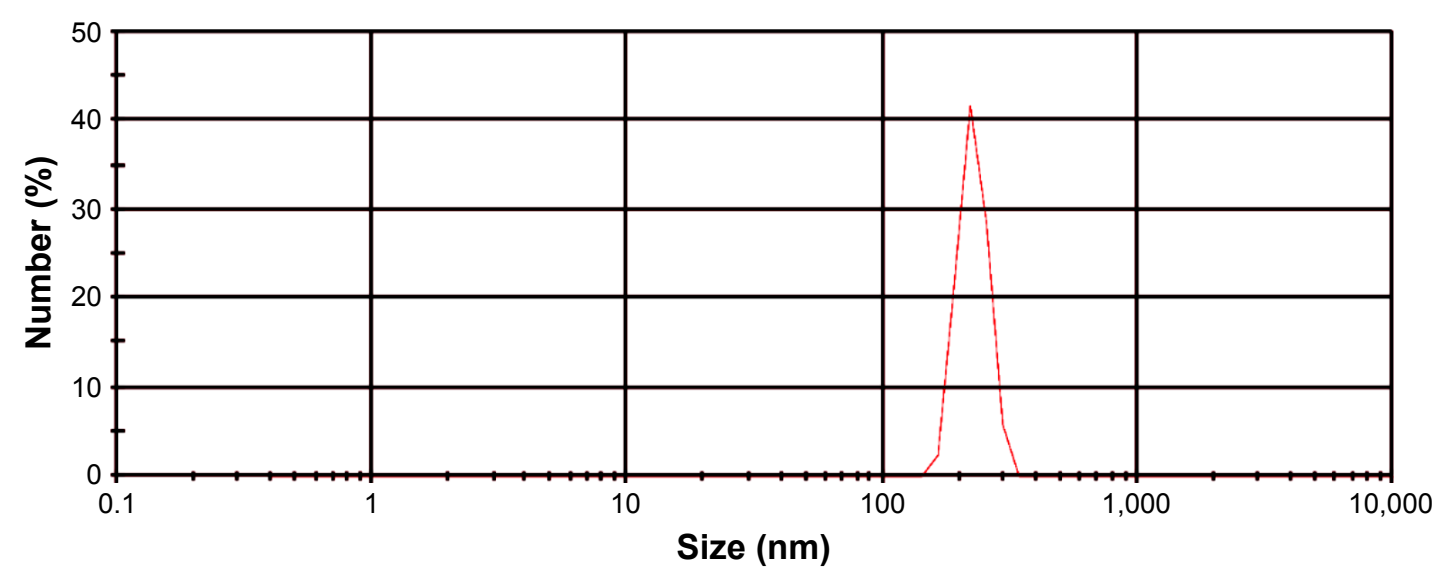

Figure 10 Particle size distribution of prepared nanocarrier by DLS technique at physiological condition $\left(\mathrm{pH} 7.4\right.$ and $37^{\circ} \mathrm{C}$ ). Abbreviation: DLS, dynamic laser scattering.

a series of pictures were snapshotted. As shown in Figure 11, the nanocarrier could easily disperse in the container.

\section{Drugs loading and release studies}

High loading drugs on the drug carrier for increasing therapeutic efficiency is an important role in the field of nanoparticulate drug-delivery systems. Regarding this issue, high-capacity drug-delivery system for loading combination anticancer drugs was designed based on amphoteric Calix. DOX and MTX were used as the model drugs with two different moieties (amine and carboxylic acid functional groups, respectively). Measurement of two important parameters is essential in drug-delivery systems including loading and encapsulation efficiencies; their calculation formula is shown below. After calculation of both parameters for both DOX and MTX, the obtained values of loading and encapsulation efficiencies for DOX were $9.02 \% \pm 2.47 \%$ and $90.22 \% \pm 5.08 \%$, respectively. However, the values of these parameters for MTX were $5.86 \% \pm 1.79 \%$ and $58.62 \% \pm 6.53 \%$, respectively. These values are related to the
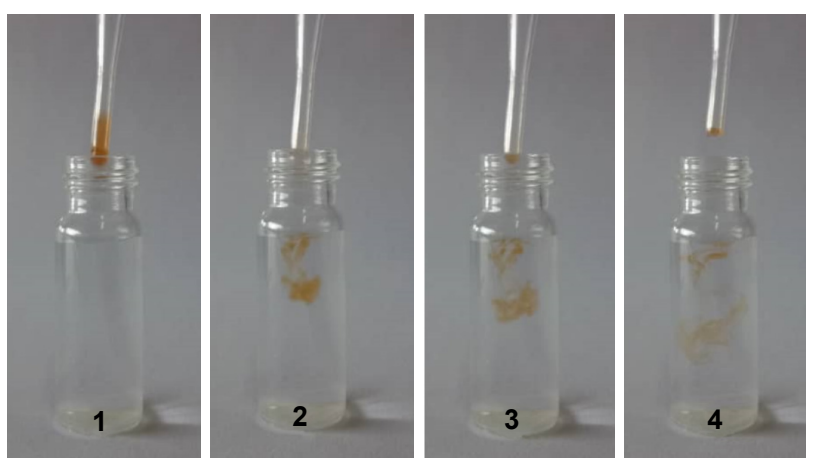

Figure I I Diffusing capability of nanocarrier in simulated physiological condition after intravenous injection ( $\mathrm{pH} 7.4$ and $\left.37^{\circ} \mathrm{C}\right)$. presence of cationic and anionic functional groups on drugs and drug carrier at the loading condition (PBS pH 7.4). The interactions are hydrogen bonding and electrostatic interaction. It is assumed that the drugs could release by changing the medium condition and causing repulsion between drugs and drug carrier. In this regard, drug release profile was simulated in both physiological and cancerous tissue conditions. DOX-MTX/nanocarrier was dispersed in PBS solution in both $\mathrm{pH} 7.4$ and 5.0, and release profiles were evaluated for 1 week (168 hours) (Figure 12). Both DOX and MTX release profiles have burst release in first and then sustain release after 1 day. The burst release of both drugs is attributed to the physical interaction between drug-carrier and drugs, so the drugs could easily separate from nanocarrier surface. After release study for 168 hours, DOX and MTX showed about $83.26 \%$ and $87.93 \%$ of cumulative releases, respectively.

\section{Biocompatibility of nanocarrier}

In biomedical applications, nanomaterials enter the body and contact with tissues and cells directly. It is essential to analyze their biocompatibility. RBCs, also called erythrocytes, represent the most abundant cellular constituent of the blood (>99\%), which plays an essential role in human health. Nanomaterials in drug-delivery systems are directly contacted with RBCs. Thus they are examined for their blood-compatible behaviors. ${ }^{38}$ Several experiments including hemolysis assay, coagulation, erythrocytes aggregations, and sedimentation rate have been carried for evaluation of biocompatibility of biomaterials before using in biomedical applications. ${ }^{34}$ In this study, hemolysis assay and erythrocytes sedimentation rate experiments as reliable and straightforward measures were chosen for estimating blood compatibility 

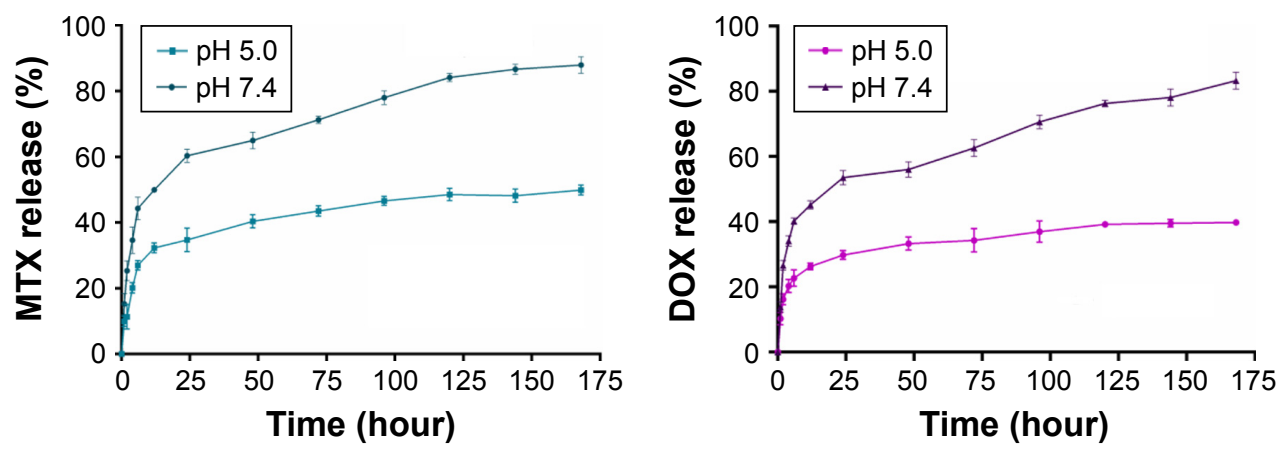

Figure 12 The cumulative release of MTX and DOX from DOX-MTX/nanocarrier at two $\mathrm{pH}$ values $(5.0$ and 7.4$)$ at $37^{\circ} \mathrm{C}$. Abbreviation: DOX-MTX/nanocarrier, DOX-MTX-loaded magnetic nanocarrier.

of materials. By treating RBCs (erythrocytes) with various concentrations of nanocarrier $(25,50,100,200,400$, and $800 \mu \mathrm{g} / \mathrm{mL}$ ), hemolytic effects were calculated using the hemolysis rate formula. As hemolysis rate has a direct relationship with the concentration of nanocarrier, a negligible hemolytic effects on RBCs was observed with $2.77 \%$ hemolysis percentage even at high treated dosage.
The results of the aggregation test on RBCs showed that nanocarrier has no effect on RBCs membrane charge and no aggregation occurred (Figure 13B). To evaluate the impact of nanocarrier on RBCs membrane charge, erythrocyte sedimentation rate (ESR) was calculated. The ESR is the rate of RBCs sedimentation in 1 hour (the ESR test is a nonspecific measure of inflammation

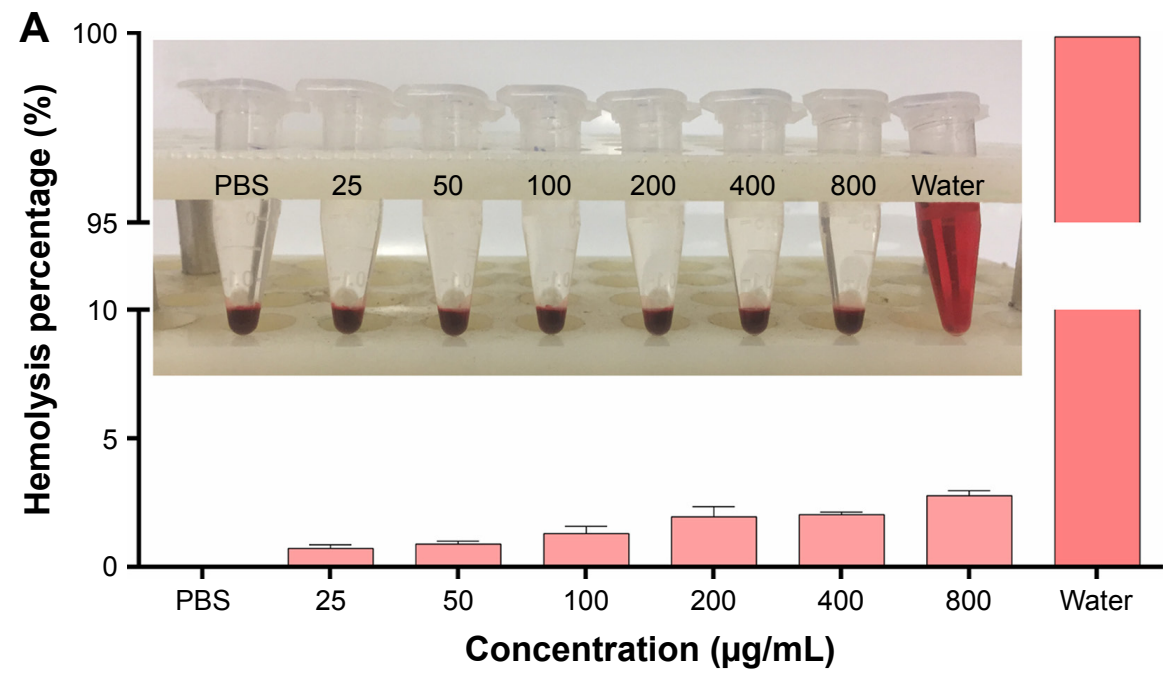

B

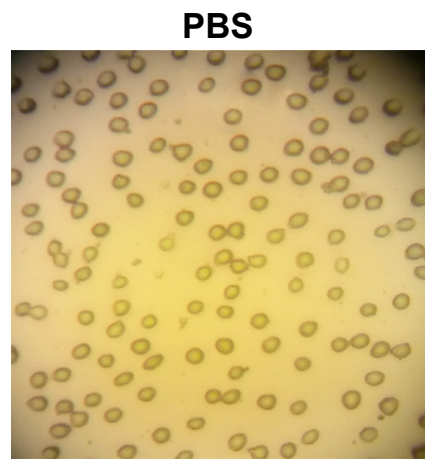

$200 \mu \mathrm{g} / \mathrm{mL}$

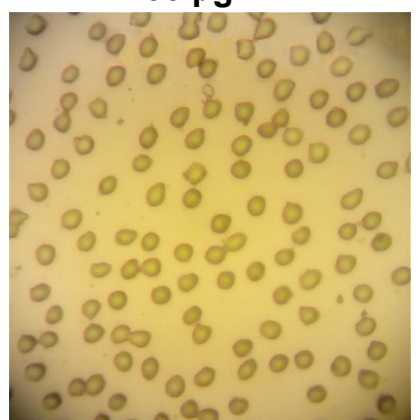

C

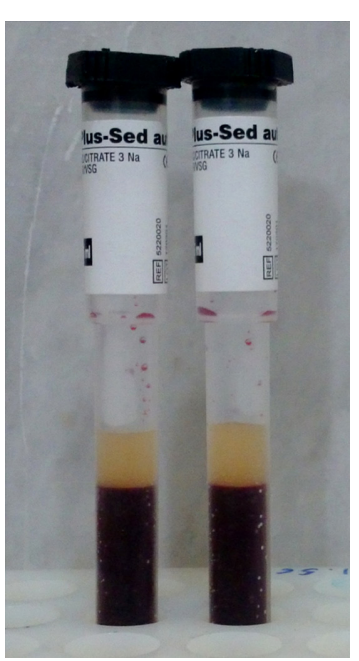

Figure 13 Three common blood compatibility analysis for determination of sample safety in blood-contacting biomedical applications. Hemolysis assay (A), RBCs aggregation test (B), and erythrocytes sedimentation rate analysis (C).

Abbreviations: PBS, phosphate buffered saline; RBCs, red blood cells. 
and hematology test). After treating RBCs with nanocarrier at the dosage of $1,000 \mu \mathrm{g} / \mathrm{mL}$ in a Westergren tube (upright tube) (containing citrate as an anticoagulant agent), the amount of sedimentation in both treated and untreated RBCs has no significant changes after 1 hour (Figure 13C). To make sure that our nanocarrier has no bad effects on the RBCs surface charge, more incubation time for interacting RBCs with nanocarrier was also done and results showed no significant difference. Ultimately, based on the results of three common blood experiments, it was expected that the developed nanocarrier is safe for using in blood-contacting biomedical applications.

\section{Cellular internalization}

The interactions of nanoparticles with the soft surfaces of biological systems such as cells play vital roles in executing their biomedical functions and in toxicity. Cells have a permeable membrane through which small molecules could easily enter into them by several pathways (concentration gradients, active transport system such as protein pumps and ion channels, and endocytosis). ${ }^{39}$ Endocytosis is a pathway that internalizes nanomaterials in transport vesicles derived from the plasma membrane. ${ }^{40}$ As our magnetic nanocarrier has a size of $<100 \mathrm{~nm}$, it could be internalized into the cells by energy-dependent clathrin-mediated endocytosis in which nanocarrier is deposited in small endocytic vesicles and fuse with early endosomes. ${ }^{41}$ To understand the nanocarrier capability in the cellular uptake process, cellular uptake was performed by fluorescent microscopy and flow cytometry analysis (two complementary methods). First, the MCF7 cells were treated with DOX-labeled nanocarrier in a timedependent manner (1, 2, and 3 hours). As shown Figure 14, all cells could uptake the DOX-labeled nanocarrier showing fluorescent light. In the next step, flow cytometry analysis was used to measure the uptake percentage and amount of fluorescent intensity (Figure 15). The results were confirmed by fluorescent microscopy observations and also showed that by increasing treating time, fluorescent intensity was enhanced. Ultimately, it could be concluded that our nanocarrier has good internalization capability into the cells, which is a crucial role for the delivery of anticancer agents into the cells.

\section{Cytotoxic assay by MTT assay}

For assessing cell metabolic activity and cytotoxicity effects of samples DOX-MTX, DOX-MTX/nanocarrier, and blank nanocarrier on MCF7 breast cancer cells, the MTT assay was performed for 48 hours (Figure 16). Before comparing the cytotoxic effects of anticancer drugs in free and nanoformulation form, the MCF7 cells (considered as model cells) were treated with blank nanocarrier to be sure for nanocarrier biocompatibility. The results revealed that nanocarrier has low cytotoxic effects on cells in the concentration range of $2-128 \mu \mathrm{g} / \mathrm{mL}$ equivalent to $99.76 \%-92.71 \%$ cell viability in a dose-dependent manner. By increasing the nanocarrier concentration, the cell viability was decreased, which attributed to the sedimentation of nanocarrier on attached

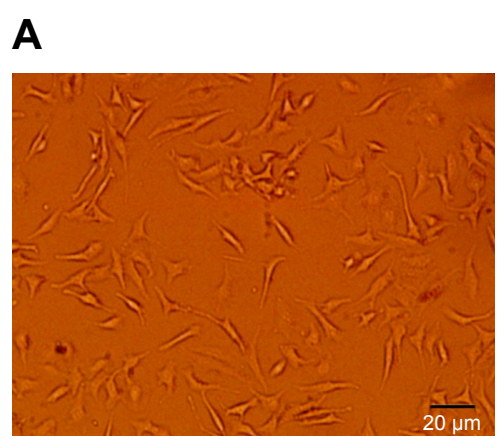

\section{B}
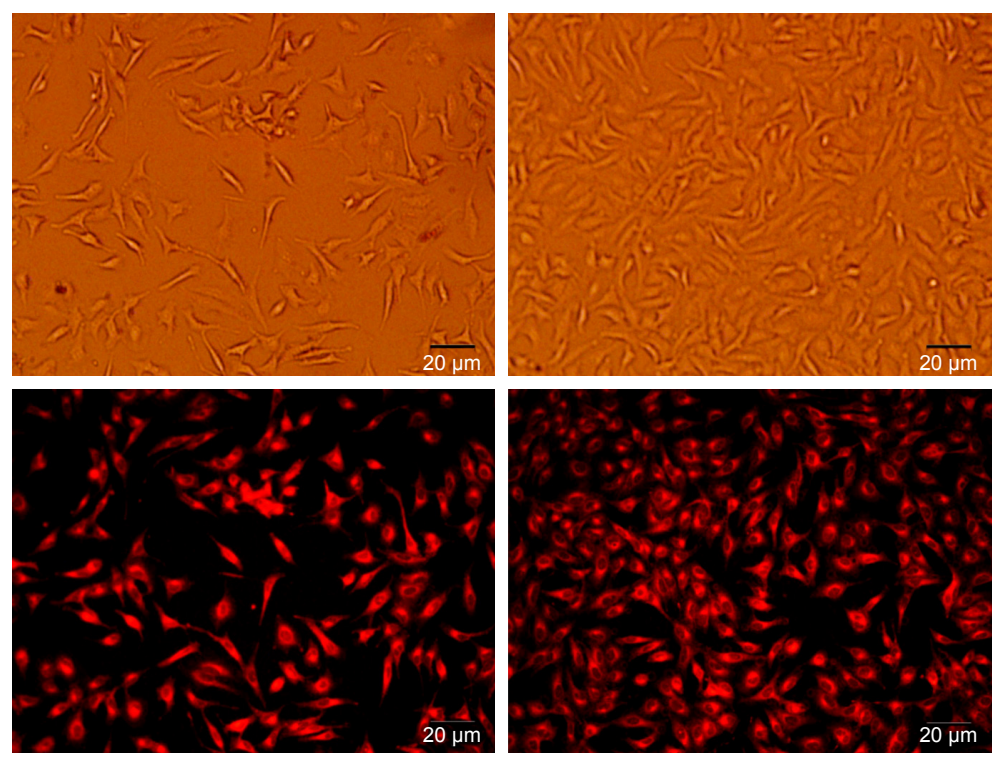

C
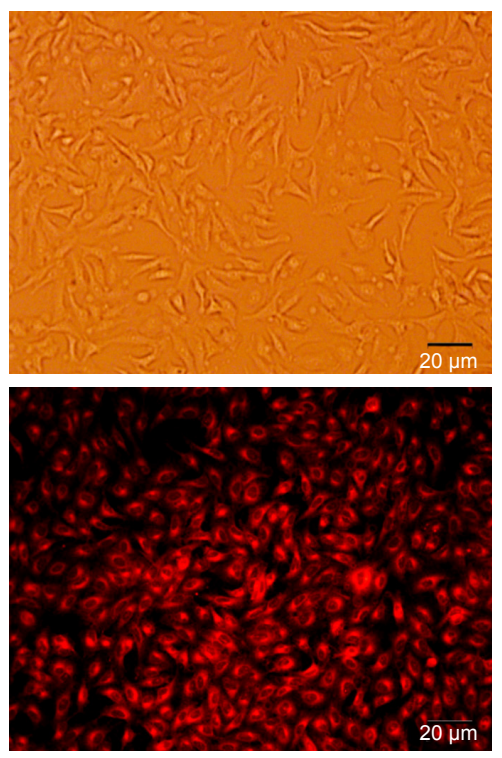

Figure 14 Cellular uptake of DOX-labeled nanocarrier by MCF7 cells after treatment for I (A), 2 (B), and 3 hours (C) by fluorescent microscopy. 


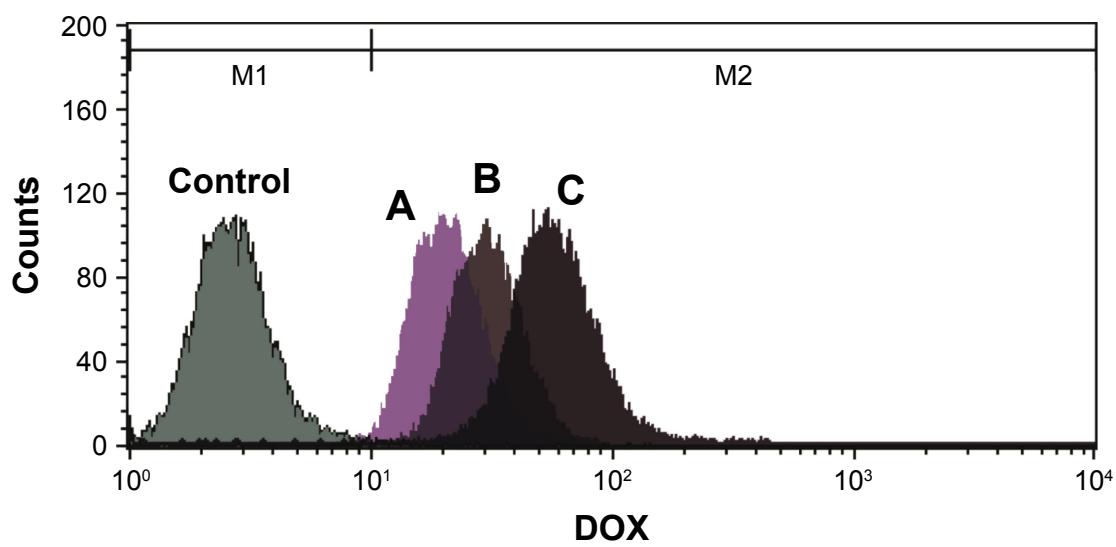

Figure 15 Cellular uptake percentage by flow cytometry after treatment for I (A), 2 (B), and 3 hours (C).

cells in the wells and causing hypoxia (deficiency in the amount of oxygen reaching the tissues). The cytotoxic effects of DOX and MTX on MCF7 cells were evaluated, and their inhibition concentration $\left(\mathrm{IC}_{50}\right)$ was measured and reported (the $\mathrm{IC}_{50}$ of both drugs are 7.22 and $9.52 \mu \mathrm{g} / \mathrm{mL}$, respectively). The cell treated with DOX-MTX/nanocarrier has low viability in comparison to DOX-MTX, and it could be related to the capability of nanocarrier internalization into the cells and releasing the drugs with control manner. Free drugs can be quickly pumped out by drug efflux transporters, which cause limitations for successful cancer chemotherapy (Figure 17). However, using drugs in nanoformulation form overcomes the transporter-mediated multidrug resistance due to the passing through of nanocarrier from the cellular membrane and releasing drugs in a controlled manner.
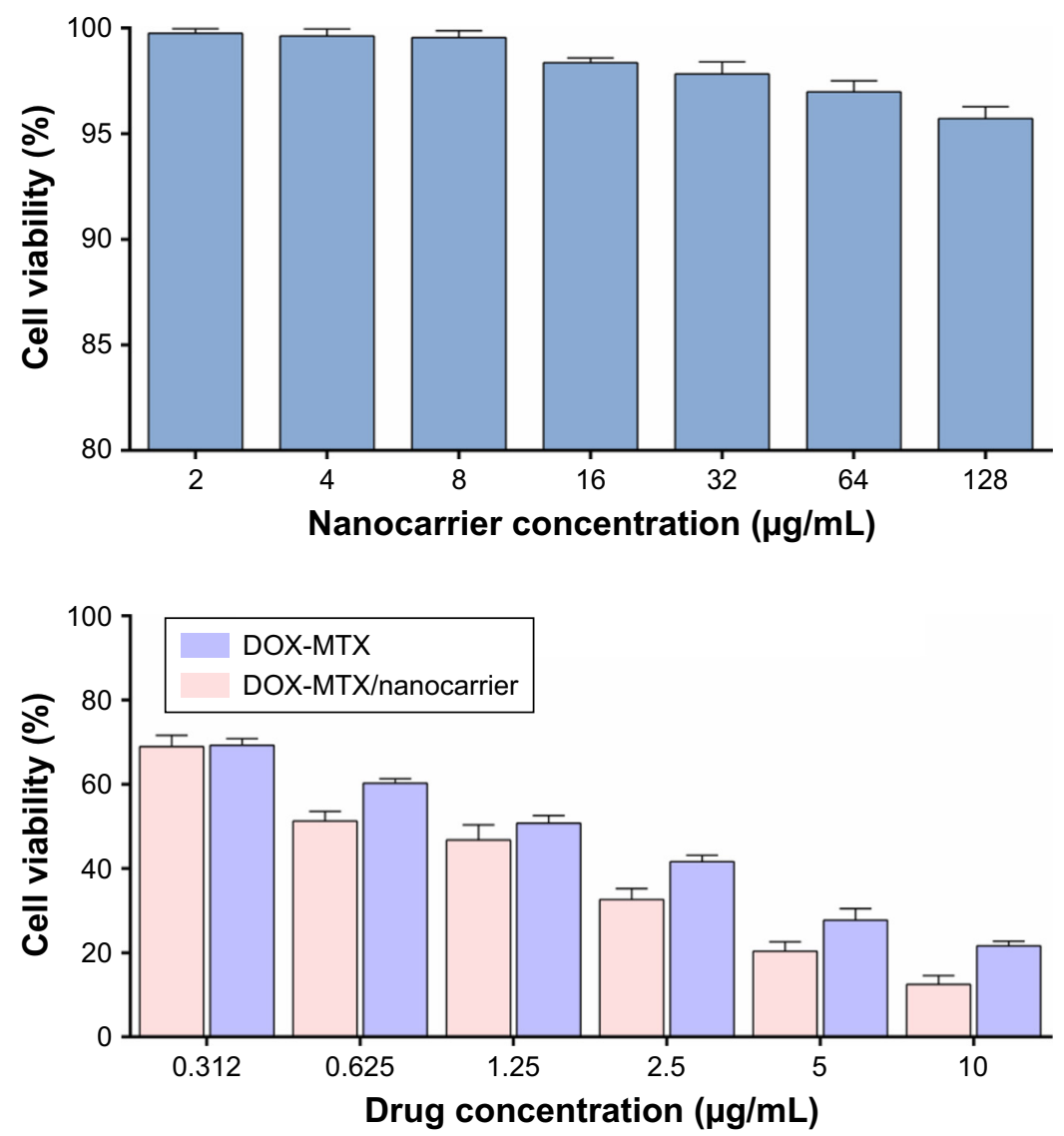

Figure 16 MTT assay for evaluation of cytotoxic effects of DOX-MTX, DOX-MTX/nanocarrier and blank nanocarrier on MCF7 breast cancer cells for 48 hours. Abbreviation: DOX-MTX/nanocarrier, DOX-MTX-loaded magnetic nanocarrier. 


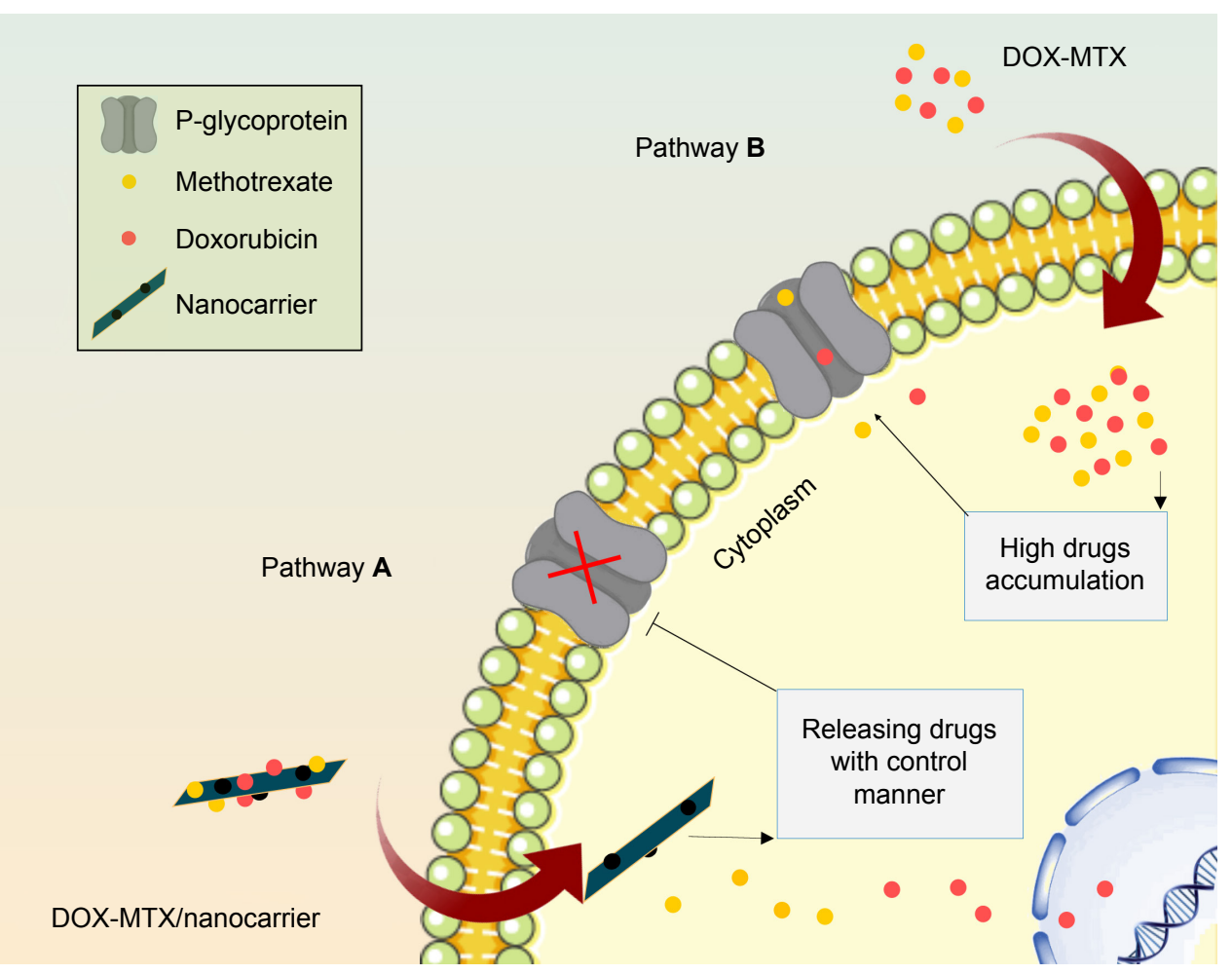

Figure 17 Drug-loaded nanocarrier internalization into the cells and releasing the drugs with control manner (Pathway A). Easily pumping out of free drugs by drug efflux transporters with low cytotoxic effects (Pathway B).

Based on ANOVA test results, there are significant differences between and within groups (DOX-MTX/nanocarrier and free DOX-MTX groups) with a $P$-value $<0.001$. The post hoc Tukey's analysis was also performed and results showed that there are significant differences with $P$-value $<0.001$, except some dosages that showed $P$-value $>0.05$ ( 1 with 7 ), (3 and 8), (4 and 9), (5 and 10), and (6 and 11) (Table 2).

\section{Apoptotic effect evaluation by DAPI staining and flow cytometry}

DAPI is a common nuclear and chromosome counterstain, which emits blue fluorescence upon binding to AT regions of
DNA. According to the DAPI characteristic, the nucleus of MCF7 cells treated with blank nanocarrier, DOX-MTX, and DOX-MTX/nanocarrier was stained with DAPI and observed under fluorescent microscopy. To compare the nuclear morphological changes of cells, the cells with no treatment considered as a control. The normal nucleus of cells has a size within a defined range, while changes in nuclear size and shape are associated with a variety of reasons. As shown in Figure 18A and B, the cells of control and treated groups with blank nanocarrier have an entire nuclear structure with normal morphology and different size of the nucleus. After treating cells with blank nanocarrier, the population of cells is

Table 2 MTT test results of MCF7 cells treated with DOX-MTX/nanocarrier with $P$-value $<0.00$ I

\begin{tabular}{|l|l|l|l|l|l|}
\hline DOX-MTX group & \multicolumn{3}{l|}{ DOX-MTX/nanocarrier group } \\
\hline No & $\begin{array}{l}\text { Drug treating } \\
\text { dose }\end{array}$ & $\begin{array}{l}\text { Cell } \\
\text { viability } \pm \text { SD }\end{array}$ & No & $\begin{array}{l}\text { Drug treating } \\
\text { dose }\end{array}$ & $\begin{array}{l}\text { Cell } \\
\text { viability } \pm \text { SD }\end{array}$ \\
\hline 1 & 0.312 & $69.270 \pm 1.549$ & 7 & 0.312 & $68.936 \pm 2.679$ \\
\hline 2 & 0.625 & $60.299 \pm 1.016$ & 8 & 0.625 & $51.299 \pm 2.227$ \\
\hline 3 & 1.250 & $50.779 \pm 1.762$ & 9 & 1.250 & $46.779 \pm 3.609$ \\
\hline 4 & 2.500 & $41.609 \pm 1.537$ & 10 & 2.500 & $32.609 \pm 2.620$ \\
\hline 5 & 5.000 & $27.733 \pm 2.758$ & 11 & 5.000 & $20.399 \pm 2.208$ \\
\hline 6 & 10.000 & $21.606 \pm 1.113$ & 12 & 10.000 & $12.458 \pm 2.115$ \\
\hline
\end{tabular}

Abbreviation: DOX-MTX/nanocarrier, DOX-MTX-loaded magnetic nanocarrier. 

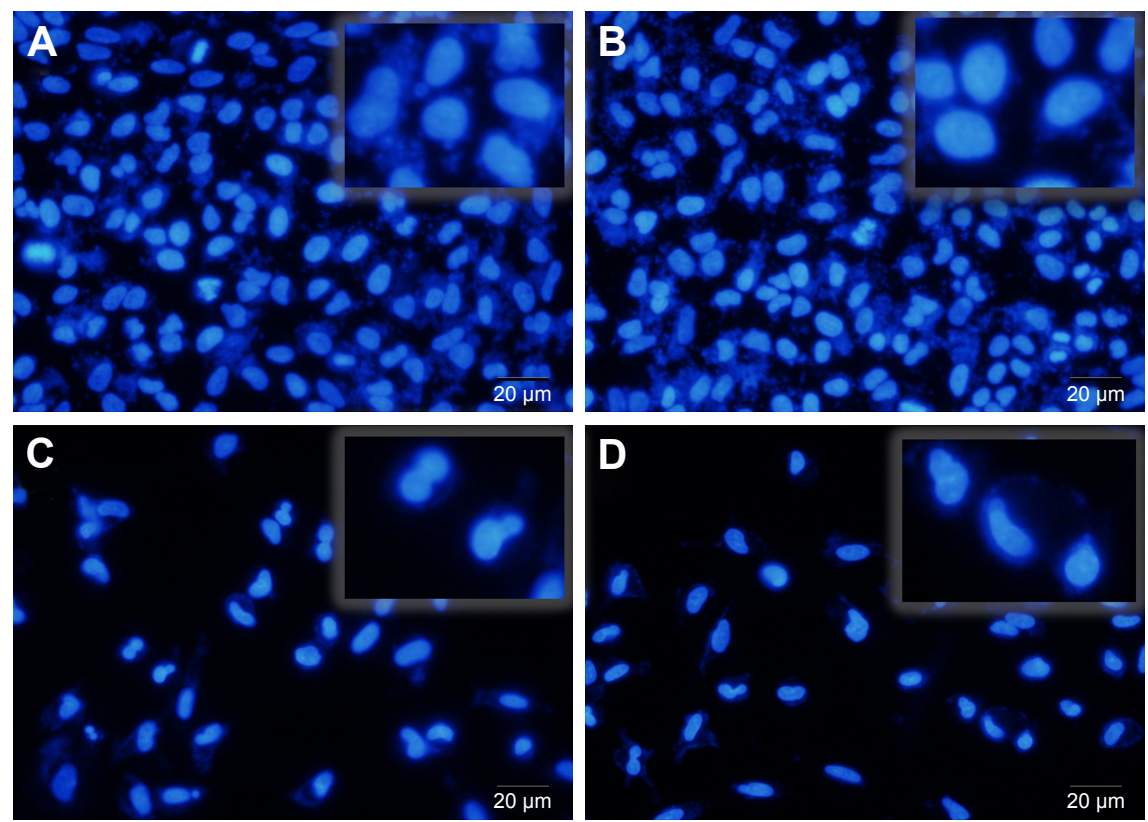

Figure 18 Fluorescent microscopic images of DAPI-stained cells following 48-hour treatment with fresh culture medium as a control (A), blank nanocarrier (B), free DOXMTX (C), and DOX-MTX/nanocarrier (D).

Abbreviation: DOX-MTX/nanocarrier, DOX-MTX-loaded magnetic nanocarrier.

A

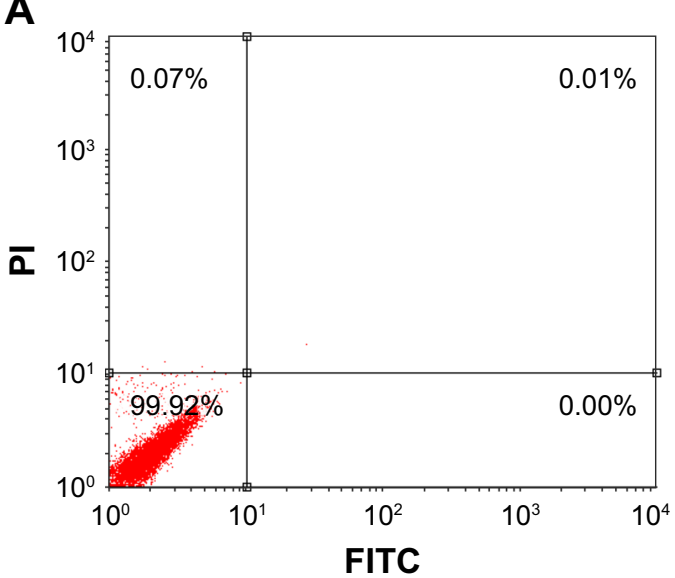

C

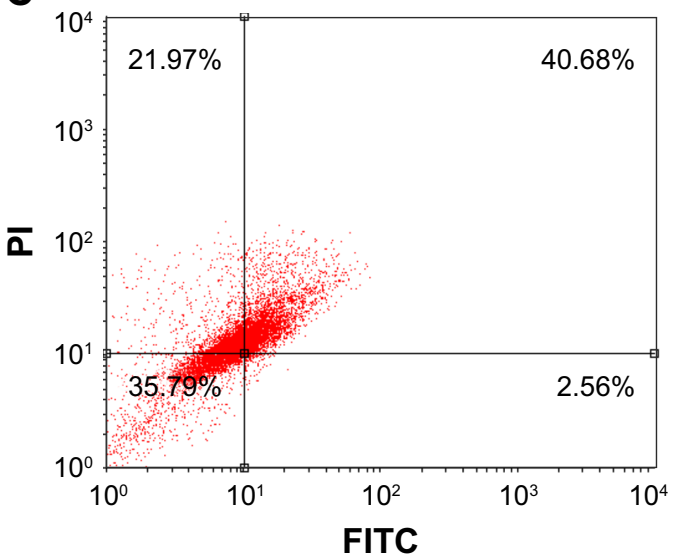

B

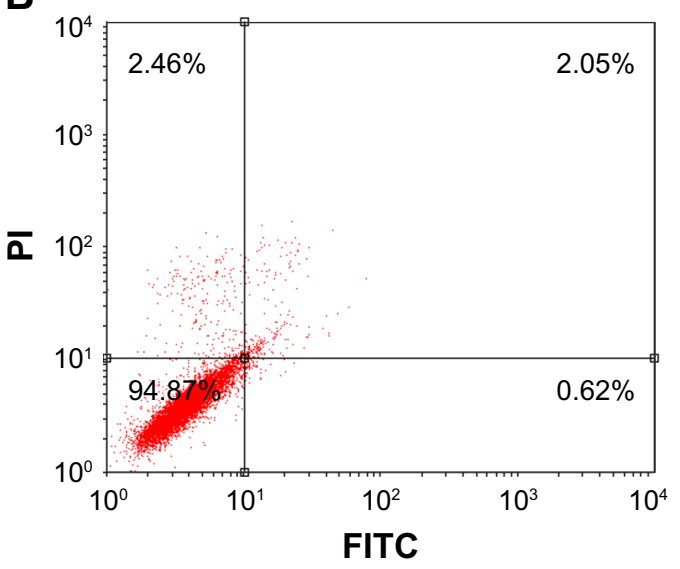

D

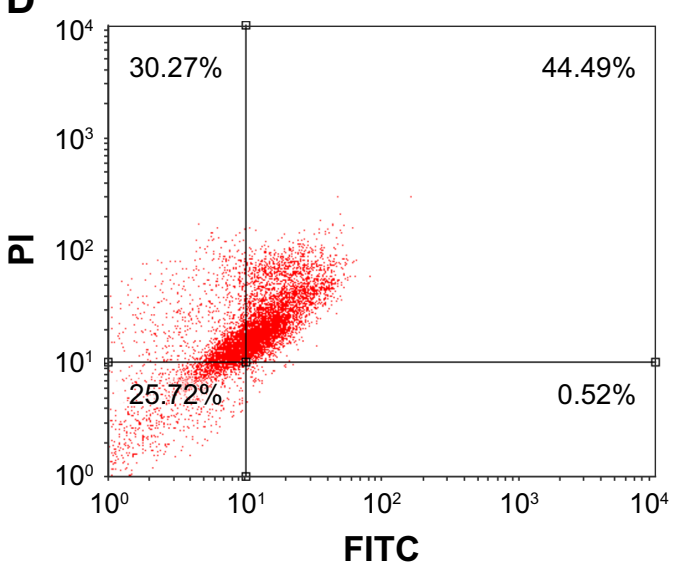

Figure 19 The apoptotic effects of MCF7 cells, determined by flow cytometry, for untreated cells as a control (A), blank nanocarrier (B), free DOX-MTX (C), and DOXMTX/nanocarrier (D).

Abbreviation: DOX-MTX/nanocarrier, DOX-MTX-loaded magnetic nanocarrier. 
the same as control. Whereas the cells treated with free drugs and drug-loaded nanocarrier have shown significant morphological changes with nuclear fragmentation, condensation, and nucleolus pyknosis (Figure 18C and D). The results showed that the formulated drug with nanocarrier has the apoptotic effects on the treated cells such as the chemotherapeutic agents.

The MCF7 cells were stained with annexin V and PI for apoptosis analysis after treatment with free DOX-MTX, DOX-MTX/nanocarrier, and blank nanocarrier for 48 hours. The cells with no treatment were considered as the control group. Based on the flow cytometric analysis, blank nanocarrier causes death of some cells because of hypoxia effects of sedimentation nanocarriers on the cells, and results suggest that the nanocarrier is safe to use in drug-delivery systems. Furthermore, the apoptotic effects of the DOXMTX/nanocarrier and free DOX-MTX were examined with a higher apoptosis rate for the drug-loaded nanocarrier in comparison to the free drugs. As shown in Figure 19, the apoptosis percentage of treated cells was reported.

\section{Conclusion}

A needle-shaped Calix with amphoteric feature was synthesized by a green method for codelivery of DOX and MTX to the MCF7 cancer cells. The developed nanocarrier has high stability with compatibility to the RBCs and human body condition. Both drugs were loaded on the nanocarrier, and the biological assays were performed to show its ability in a biomedical application such as drug-delivery systems. Three blood compatibility tests were examined, and results showed that designed nanocarrier has low hemolytic effects, erythrocytes sedimentation, and aggregation. Cellular uptake by fluorescent microscopy and flow cytometry analysis showed that nanocarrier could internalize into the cells easily in the first treatment.

Regarding the high internalization of nanocarrier into the cells, the cytotoxicity assay was performed, and the results showed that drug-loaded nanocarrier has good efficacy in killing cells, and this is related to the drugs releasing with control and sustained manner. Other assays such as DAPI staining and annexin V and PI were performed to show the apoptotic effect of drug-loaded nanocarrier in comparison to the free drugs. As a result, the developed nanocarrier is a promising effective vehicle for the codelivery of therapeutic agents and has excellent capability to deliver other biological agents such as genes and antibiotics.

\section{Acknowledgment}

The authors would like to thank the Drug Applied Research Center, Tabriz University of Medical Sciences, Tabriz, Iran (all experiments in this study were done in this research center).

\section{Disclosure}

The authors report no conflicts of interest in this work.

\section{References}

1. Wong BS, Yoong SL, Jagusiak A, et al. Carbon nanotubes for delivery of small molecule drugs. Adv Drug Deliv Rev. 2013;65(15):1964-2015.

2. Rahimi M, Shojaei S, Safa KD, et al. Biocompatible magnetic tris(2-aminoethyl)amine functionalized nanocrystalline cellulose as a novel nanocarrier for anticancer drug delivery of methotrexate. New $J$ Chem. 2017;41(5):2160-2168.

3. Rahimi M, Shafiei-Irannejad V, D Safa K, Salehi R. Multi-branched ionic liquid-chitosan as a smart and biocompatible nano-vehicle for combination chemotherapy with stealth and targeted properties. Carbohydr Polym. 2018;196:299-312.

4. Salehi R, Davaran S, Rashidi MR, Entezami AA. Thermosensitive nanoparticles prepared from poly $(N$-isopropylacrylamide-acrylamidevinilpyrrolidone) and its blend with poly(lactide-co-glycolide) for efficient drug delivery system. J Appl Polym Sci. 2009;111(4): $1905-1910$.

5. Wang K, Guo DS, Wang X, Liu Y. Multistimuli responsive supramolecular vesicles based on the recognition of $p$-Sulfonatocalixarene and its controllable release of doxorubicin. ACS Nano. 2011;5(4):2880-2894.

6. Rahimi M, Karimian R, Mostafidi E, et al. Highly branched aminefunctionalized $p$-sulfonatocalix[4]arene decorated with human plasma proteins as a smart, targeted, and stealthy nano-vehicle for the combination chemotherapy of MCF7 cells. New J Chem. 2018;42(15):13010-13024.

7. Hu QD, Tang GP, Chu PK. Cyclodextrin-based host-guest supramolecular nanoparticles for delivery: from design to applications. Acc Chem Res. 2014;47(7):2017-2025.

8. Ma D, Hettiarachchi G, Nguyen D, et al. Acyclic cucurbit[n]uril molecular containers enhance the solubility and bioactivity of poorly soluble pharmaceuticals. Nat Chem. 2012;4(6):503-510.

9. Ghosh P, Khan TK, Bharadwaj PK. Cryptand-based metal-free or complexed amphiphiles which readily form vesicles. Chem Comm. 1996; 2(2):189-190.

10. Zhang M, Yan X, Huang F, Niu Z, Gibson HW. Stimuli-responsive host-guest systems based on the recognition of cryptands by organic guests. Acc Chem Res. 2014;47(7):1995-2005.

11. Lee SF, Zhu XM, Wang YX, et al. Ultrasound, $\mathrm{pH}$, and magnetically responsive crown-ether-coated core/shell nanoparticles as drug encapsulation and release systems. ACS Appl Mater Interfaces. 2013;5(5): $1566-1574$.

12. Asfari M-Z, Böhmer V, Harrowfield J, Vicens J. Calixarenes 2001. Dordrecht: Kluwer Academic Publishers; 2001.

13. Ede J, Cragg P, Sambrook M. Comparison of binding affinities of watersoluble calixarenes with the organophosphorus nerve agent soman $(\mathrm{Gd})$ and commonly-used nerve agent simulants. Molecules. 2018;23(1):207.

14. Sharma VS, Sharma AS, Vekariya RH. Columnar self-assembly of bowl shaped fluorescent liquid crystals based on calix[4]arene with Schiff base units. New J Chem. 2018;42(18):15044-15051.

15. Muneer S, Memon S, Pahnwar QK, Bhatti AA, Khokhar TS. Synthesis and investigation of antimicrobial properties of pyrrolidine appended calix[4]arene. J Anal Sci Technol. 2017;8(1):3.

16. Zhu JM, Chen LX, Chen K, Zeng X, Tao Z. Synthesis of a functionalised calix[4] arene and its interactions with hemicucurbit[6,7]urils and cucurbit[8]uril. Tetrahedron. 2018;74(30):4095-4099.

17. Ma X, Zhao Y. Biomedical applications of supramolecular systems based on host-guest interactions. Chem Rev. 2015;115(15): 7794-7839.

18. Zakerzadeh E, Alizadeh E, Samadi Kafil H, Mohammad Hassanzadeh A, Salehi R, Mahkam M. Novel antibacterial polymeric nanocomposite for smart co-delivery of anticancer drugs. ArtifCells Nanomed Biotechnol. 2017;45(8):1509-1520.

19. Farshbaf M, Salehi R, Annabi N, Khalilov R, Akbarzadeh A, Davaran S. $\mathrm{pH}-$ and thermo-sensitive MTX-loaded magnetic nanocomposites: synthesis, characterization, and in vitro studies on A549 lung cancer cell and MR imaging. Drug Dev Ind Pharm. 2018;44(3):452-462. 
20. Farzi-Khajeh H, Safa KD, Dastmalchi S. Arsanilic acid modified superparamagnetic iron oxide nanoparticles for purification of alkaline phosphatase from hen's egg yolk. J Chromatography B. 2017;1061:26-33.

21. Marszałł MP. Application of magnetic nanoparticles in pharmaceutical sciences. Pharm Res. 2011;28(3):480-483.

22. Shafiei-Irannejad V, Samadi N, YousefiB, Salehi R, Velaei K, Zarghami N. Metformin enhances doxorubicin sensitivity via inhibition of doxorubicin efflux in P-gp-overexpressing MCF-7 cells. Chem Biol Drug Des. 2018;91(1):269-276.

23. Shafiei-Irannejad V, Samadi N, Salehi R, et al. Reversion of multidrug resistance by co-encapsulation of doxorubicin and metformin in Poly(lactide-co-glycolide)-d- $\alpha$-tocopheryl polyethylene glycol 1000 succinate nanoparticles. Pharm Res. 2018;35(6):119.

24. Rahimi M, Safa KD, Salehi R. Co-delivery of doxorubicin and methotrexate by dendritic chitosan-g-mPEG as a magnetic nanocarrier for multidrug delivery in combination chemotherapy. Polymer Chem. 2017;8(47): 7333-7350.

25. Rahimi M, Safa KD, Alizadeh E, Salehi R. Dendritic chitosan as a magnetic and biocompatible nanocarrier for the simultaneous delivery of doxorubicin and methotrexate to MCF-7 cell line. New J Chem. 2017; 41(8):3177-3189.

26. Shafiei-Irannejad V, Samadi N, Salehi R, Yousefi B, Zarghami N. New insights into antidiabetic drugs: possible applications in cancer treatment. Chem Biol Drug Des. 2017;90(6):1056-1066.

27. Yang Z, Chang L, Chiang CL, Lee LJ, James Lee L. Micro-/nanoelectroporation for active gene delivery. Curr Pharm Des. 2015;21(42): 6081-6088.

28. Kavianinia I, Plieger PG, Kandile NG, Harding DRK. Preparation and characterization of an amphoteric chitosan derivative employing trimellitic anhydride chloride and its potential for colon targeted drug delivery system. Mater Today Commun. 2015;3:78-86.

29. Tong Y, Ganbold T, Baigude H. Synthesis of amphoteric curdlan derivatives for delivery of therapeutic nucleic acids. Carbohydr Polym. 2017;175:739-745.

30. Omer AM, Tamer TM, Hassan MA, Rychter P, Mohy Eldin MS, Koseva N. Development of amphoteric alginate/aminated chitosan coated microbeads for oral protein delivery. Int J Biol Macromol. 2016; 92:362-370.
31. Moorthy MS, Park J-H, Bae J-H, Kim S-H, Ha C-S. Mesoporous organosilica hybrids with a tunable amphoteric framework for controlled drug delivery. J Mater Chem B. 2014;2(38):6487-6499.

32. Xiong D, Chen M, Li H. Synthesis of para-sulfonatocalix[4]arenemodified silver nanoparticles as colorimetric histidine probes. Chem Commun. 2008;45(7):880-882.

33. Xue Y, Guan Y, Zheng A, Xiao H. Amphoteric calix[8]arene-based complex for $\mathrm{pH}$-triggered drug delivery. Colloids Surf B Biointerfaces. 2013;101:55-60.

34. Huang H, Lai W, Cui M, et al. An evaluation of blood compatibility of silver nanoparticles. Sci Rep. 2016;6(1):25518.

35. Agotegaray MA, Campelo AE, Zysler RD, et al. Magnetic nanoparticles for drug targeting: from design to insights into systemic toxicity. preclinical evaluation of hematological, vascular and neurobehavioral toxicology. Biomater Sci. 2017;5(4):772-783.

36. Jabbari S, Ghamkhari A, Javadzadeh Y, Salehi R, Davaran S. Doxorubicin and chrysin combination chemotherapy with novel $\mathrm{pH}$-responsive poly [(lactide-co-glycolic acid)-block-methacrylic acid] nanoparticle. J Drug Deliv Sci Technol. 2018;46:129-137.

37. Abbasian M, Mahmoodzadeh F, Salehi R, Amirshaghaghi A. Chemophotothermal therapy of cancer cells using gold nanorod-cored stimuliresponsive triblock copolymer. New JChem. 2017;41(21):12777-12788.

38. Li X, Wang L, Fan Y, Feng Q, Cui F-Z. Biocompatibility and toxicity of nanoparticles and nanotubes. J Nanomater. 2012;2012(4):1-19.

39. Zhao F, Zhao Y, Liu Y, Chang X, Chen C, Zhao Y. Cellular uptake, intracellular trafficking, and cytotoxicity of nanomaterials. Small. 2011; 7(10):1322-1337.

40. Riehemann K, Schneider SW, Luger TA, Godin B, Ferrari M, Fuchs H. Nanomedicine - challenge and perspectives. Angew Chem Int Ed Engl. 2009;48(5):872-897.

41. Li W, Chen C, Ye C, et al. The translocation of fullerenic nanoparticles into lysosome via the pathway of clathrin-mediated endocytosis. Nanotechnology. 2008;19(14):145102.
International Journal of Nanomedicine

\section{Publish your work in this journal}

The International Journal of Nanomedicine is an international, peerreviewed journal focusing on the application of nanotechnology in diagnostics, therapeutics, and drug delivery systems throughout the biomedical field. This journal is indexed on PubMed Central, MedLine, CAS, SciSearch $®$, Current Contents ${ }^{\circledR} /$ Clinical Medicine,

\section{Dovepress}

Journal Citation Reports/Science Edition, EMBase, Scopus and the Elsevier Bibliographic databases. The manuscript management system is completely online and includes a very quick and fair peer-review system, which is all easy to use. Visit http://www.dovepress.com/ testimonials.php to read real quotes from published authors. 